Accepted to ApJ: August 4, 2009

Preprint typeset using LATEX style emulateapj v. 08/22/09

\title{
COMPACT STAR CLUSTERS IN THE M31 DISK
}

\author{
V. VAnsevičius ${ }^{1,2}$, K. Kodaira ${ }^{3}$, D. NArbutis ${ }^{1,2,4}$, R. Stonkuté ${ }^{1}$, \\ A. Bridžius ${ }^{1}$, V. Deveikis ${ }^{2}$, And D. Semionov ${ }^{1}$ \\ AcCePted to ApJ: August 4, 2009
}

\begin{abstract}
We have carried out a survey of compact star clusters (apparent size $\lesssim 3^{\prime \prime}$ ) in the southwest part of the M31 galaxy, based on the high-resolution Suprime-Cam images $\left(17.5^{\prime} \times 28.5^{\prime}\right)$, covering $\sim 15 \%$ of the deprojected galaxy disk area. The $U B V R I$ photometry of 285 cluster candidates $(V \lesssim 20.5 \mathrm{mag})$ was performed using frames of the Local Group Galaxies Survey. The final sample, containing 238 high probability star cluster candidates (typical half-light radius $r_{h} \sim 1.5 \mathrm{pc}$ ), was selected by specifying a lower limit of $r_{h} \gtrsim 0.15^{\prime \prime}(\gtrsim 0.6 \mathrm{pc})$. We derived cluster parameters based on the photometric data and multiband images by employing simple stellar population models. The clusters have a wide range of ages from $\sim 5 \mathrm{Myr}$ (young objects associated with $24 \mu \mathrm{m}$ and/or $H \alpha$ emission) to $\sim 10$ Gyr (globular cluster candidates), and possess mass in a range of $3.0 \lesssim \log \left(m / m_{\odot}\right) \lesssim 4.3$ peaking at $m \sim 4000 m_{\odot}$. Typical age of these intermediate-mass clusters is in the range of $30 \mathrm{Myr} \lesssim t \lesssim 3 \mathrm{Gyr}$, with a prominent peak at $\sim 70 \mathrm{Myr}$. These findings suggest a rich intermediate-mass star cluster population in M31, which appears to be scarce in the Milky Way galaxy.
\end{abstract}

Subject headings: galaxies: individual (M31) - galaxies: star clusters - stars: formation

\section{INTRODUCTION}

The Andromeda galaxy, M31, as the nearest spiral galaxy, has been regarded to be the most suitable to provide supplementary data to the Galactic data for understanding the galaxy structures, and to further elucidate the evolution history of disk galaxies. Since a star cluster normally represents a single population, many detailed studies of star clusters in M31 were devoted to deriving information related to the structure and evolution of star clusters and of hosting galaxy: see the review in Kodaira (2002) and the introduction of Caldwell et al. (2009), and the works cited therein, among others.

Recently, many observational works were devoted to study globular clusters (GCs; see, e.g., Barmby et al. 2002, 2007, and references therein). The study by Cohen et al. (2005), based on high-resolution Keck imaging, however, suggests that M31 cluster samples (e.g., Galleti et al. 2007) might be heavily contaminated by asterisms. Nevertheless, the discussion on this issue by Caldwell et al. (2009) states that appearance of lowmass clusters dominated by several bright stars depends strongly on the photometric passband used, and emphasizes the need of multiband observations to identify clusters correctly.

M31 disk cluster studies benefited from the highresolution Hubble Space Telescope (HST) images, which were used by Williams \& Hodge (2001a,b) to identify young star cluster candidates. Recently an extensive archival BVI image survey of $H S T$ WFPC2/ACS instrument fields, which are scattering over a wide area of M31 in patches, was conducted in search of new clus-

\footnotetext{
${ }^{1}$ Institute of Physics, Savanoriug 231, Vilnius LT-02300, Lithuania; donatas.narbutis@ff.vu.lt

${ }^{2}$ Vilnius University Observatory, Čiurlionio 29, Vilnius LT03100, Lithuania

3 The Graduate University for Advanced Studies (SOKENDAI), Shonan Village, Hayama, Kanagawa 240-0193, Japan

${ }^{4}$ Author to whom any correspondence should be addressed.
}

ters (see Krienke \& Hodge 2007, 2008, and references therein).

However, until the recent years, when high-resolution wide-field images became available, much less efforts have been devoted for a homogeneous wide-field photometric survey of objects, which have properties in-between of globular and open clusters. Our survey, using SuprimeCam (Miyazaki et al. 2002) mosaic images, completely covers the area of $17.5^{\prime} \times 28.5^{\prime}$ in the southwestern part of the M31 disk (see Kodaira et al. 2004). A dozen of $H S T$ frames are located in our field covering less than $20 \%$.

Our initial study of M31 compact clusters of an apparent size less than $\sim 3^{\prime \prime}$ using Suprime-Cam revealed that some of the previously suspected GC candidates should be classified as open clusters (OCs), suggesting that there is a new kind of star cluster, which is not well known in the Milky Way galaxy (MW), but whose counterparts are probably present in Large Magellanic Cloud/Small Magellanic Cloud (LMC/SMC; Kodaira 2002; Kodaira et al. 2004, 2008). They are apparently more massive than typical OCs in the solar neighborhood (Piskunov et al. 2008), but less massive than the typical GCs in MW (McLaughlin \& van der Marel 2005). This suggests the presence of an intermediate disk population of star clusters, a detailed study of which may contribute to the understanding of disk evolution history. For examples of the typical images of compact clusters, see the atlas published by Kodaira et al. (2004).

About 50 bright $(17.0 \lesssim V \lesssim 19.0 \mathrm{mag})$ members of our M31 cluster sample (Narbutis et al. 2006) were studied in detail concerning their radial surface brightness profiles using a tidal-cutoff King model (King 1962) and a power-law EFF model (Elson et al. 1987). Their distribution in the half-light radius versus the absolute $V$-band magnitude diagram (Sablevičiūtè et al. 2007) shows that these compact clusters are occupying the same domain as faint GCs (e.g., Barmby et al. 2007). 
A comparison in the parameter domain of the King model (Sablevičiūtè et al. 2006) confirms a visual impression that compact clusters have similar radial profiles to GCs, except for minor irregularities, which become visible in high-resolution images. Comparison of the EFF model parameters of compact clusters and massive clusters in LMC (Mackey \& Gilmore 2003) and SMC (Hill \& Zaritsky 2006) shows that the present sample of compact clusters may have the same structural nature as massive clusters in the Magellanic Clouds (Sablevičiūtè et al. 2006).

These promising results motivated us to extend the survey of clusters to a deeper magnitude of $V \lesssim$ $20.5 \mathrm{mag}$. The homogeneous $U B V R I$ photometric data and multiband images for 285 selected cluster candidates with absolute magnitudes down to $M_{V} \lesssim-4$ mag were analyzed in the catalog paper by Narbutis et al. (2008). Here, we present the results of simple stellar population (SSP) model fitting using the multiband photometry data, giving mass and age of these objects in the surveyed field of M31.

We have developed and applied a method to derive structural parameters from the surface brightness distribution of clusters by properly accounting for the contaminating stars, superposed on their smooth profile. The derived half-light radii were studied in connection with the SSP model fitting results. We have also modeled stochastic effects of bright stars (Deveikis et al. 2008) and briefly discuss their influence on the derived star cluster parameters.

In the following, we describe observations and sample selection in Section 2 present structural and evolutionary star cluster parameters in Section 3, and analyze properties of studied sample of M31 cluster candidates in Section 4. Properties of a few particular objects are discussed in the Appendix.

\section{OBSERVATION}

\subsection{Data}

We have identified high probability star cluster candidates (hereafter star clusters) by visually inspecting the Suprime-Cam $V$-band mosaic image made from $5 \times$ 2 minute individual exposures of the $17.5^{\prime} \times 28.5^{\prime}$ field, centered at $\alpha_{\mathrm{J} 2000}=0^{\mathrm{h}} 40.9^{\mathrm{m}}, \delta_{\mathrm{J} 2000}=+40^{\circ} 45^{\prime}$. The characteristic full width at half maximum (FWHM) of stellar images (point-spread function, PSF) is $0.7^{\prime \prime}$. See Kodaira et al. (2004) for a description of the SuprimeCam observations and data reduction details.

We have produced the homogenized $U B V R I$ photometric catalog of 285 selected star clusters $(V \lesssim$ $20.5 \mathrm{mag}$ ) used as an initial sample in this study, based on photometry performed on the Local Group Galaxies Survey (LGGS) mosaic images (Massey et al. 2006). The variable PSFs of four overlapping LGGS field (F6-F9) mosaic images were homogenized to the same FWHM = $1.5^{\prime \prime}$. The mosaics were photometrically calibrated by using individual CCD color equations and the stellar photometry catalog from Massey et al. (2006) as a local photometric standard for zero point calibration. Different aperture sizes and photometric background estimation areas were selected for individual clusters, as they are given in the catalog paper (Narbutis et al. 2008).

We note that a comparison of three published stellar photometry data sets in our survey field (Narbutis et al. 2006) makes one to be cautious when using tertiary standards as local photometric standards. However, we used a carefully reduced and calibrated data set by Massey et al. (2006), which passed an internal consistency check, making it the most accurately calibrated photometry catalog of the M31 galaxy to date.

The color images of clusters were produced from the LGGS $(U, B, V, I, H \alpha), G A L E X$ (FUV, NUV), 2MASS $\left(J, H, K_{\mathrm{s}}\right)$, Spitzer $(24 \mu \mathrm{m})$, and HI $(21 \mathrm{~cm})$ images. They served as references for object properties and environment study. Seventy-seven star clusters from our catalog are located in the HST archive frames. For object selection, photometry, and multiband image details, see Narbutis et al. (2008).

We used Suprime-Cam $V$-band mosaic image $\left(\mathrm{FWHM}_{\mathrm{PSF}} \approx 0.7^{\prime \prime}\right.$, image scale $\left.0.2^{\prime \prime} / \mathrm{pixel}\right)$ to derive structural parameters of star clusters. The LGGS field's F7 $V$-band mosaic image $\left(\mathrm{FWHM}_{\mathrm{PSF}} \approx 0.8^{\prime \prime}\right.$, image scale of $0.27^{\prime \prime} /$ pixel) was substituted for objects saturated or having defects in the Suprime-Cam frame. Since the variability of $\mathrm{FWHM}_{\mathrm{PSF}}$ was only $\sim 5 \%$ across the mosaic (see Section 3.1 for details), we have applied the DAOPHOT package (Stetson 1987) from the IRAF program system (Todv 1993) to compute a single mosaic PSF. IRAF's program "seepsf" was used to create PSFs suitable for our structural parameter fitting program.

The following M31 parameters are adopted in this study: distance modulus of $m-M=24.47(785 \mathrm{kpc}$, thus $1^{\prime \prime} \equiv 3.8 \mathrm{pc}$; McConnachie et al. 2005); center coordinates $\alpha_{\mathrm{J} 2000}=0^{\mathrm{h}} 42^{\mathrm{m}} 44.3^{\mathrm{s}}, \delta_{\mathrm{J} 2000}=41^{\circ} 16^{\prime} 09^{\prime \prime}(\mathrm{NASA}$ Extragalactic Database); major axis position angle of $38^{\circ}$ (de Vaucouleurs 1958); disk inclination angle to the line of sight of $75^{\circ}$ (Gordon et al. 2006).

\subsection{Sample Selection}

The star clusters were selected from the initial sample of 285 objects by considering the following criteria: (1) the derived half-light radius is of $r_{h} \geq 0.15^{\prime \prime}(\geq 0.6 \mathrm{pc})$ this limit arises due to the resolution of ground-based observations; (2) there are no nearby contaminants strongly influencing the accuracy of the derived structural and evolutionary parameters. Note however, that several genuine star clusters, judged from the $H S T$ images, did not meet these criteria.

A few dim, i.e., low central surface brightness, objects with an unreasonably large derived half-light radius or composed of several resolved stars were rejected because of low reliability of determined parameters. Although PÉGASE (Fioc \& Rocca-Volmerange 1997) SSP models incorporate nebular emission lines for young age objects, we stress that structural and evolutionary parameters of clusters showing up $H \alpha$ emissions or superposed on inhomogeneous diffuse $H \alpha$ background should be considered with caution. Note however, that objects with strong $H \alpha$ emission were cataloged separately by Kodaira et al. (2004), therefore, these objects were excluded from the analysis in this study. We note, that foreground starburst galaxies possessing $24 \mu \mathrm{m}$ emission, which have been revealed during spectroscopic study by Caldwell et al. (2009), might be present in a sample of young $(t \lesssim 20 \mathrm{Myr}$ ) clusters (see Appendix for details on KW271 and KW279). 
This cleaning yielded a sample of 238 star clusters. Their ID numbers and center coordinates from Narbutis et al. (2008) are supplemented with the structural and evolutionary parameters described in the following, and are presented in Table 1, the full form of which is available in the electronic edition of the Journal.

\section{MODEL FITTING}

\subsection{Structural Parameters}

The studied star clusters, projected on the crowded disk of M31, are unresolved or semiresolved in the Suprime-Cam images. Background and/or clusters' bright stars superposed on clusters' surface brightness distribution strongly influence photometric (Narbutis et al. 2007b) and structural parameter accuracy. However, stars projecting nearby to the cluster cannot be simply masked out because of flux superposition in their extended wings. To avoid this, we have developed a method to derive structural parameters of star clusters by including resolved stars in the cluster model fitting procedure. The method is presented in D. Semionov et al. (2009, in preparation); here we provide its brief description and derived half-light radii of star clusters (Table 1).

The developed program tool models star clusters as a smooth analytical surface brightness distributions. Observational effects are taken into account by convolving cluster models with PSF. Individual resolved stars are modeled with PSF. Free parameters, which describe a shape and position of the cluster model, are fitted using a genetic algorithm, providing the global solution. Fine tuning of this solution is performed using LevenbergMarquardt nonlinear least-square algorithm. A photometric background value, which is kept constant during model fit, is estimated within individually selected circular annulus centered on the object. The model fitting radius is chosen seeking to enclose extended wings of the surface brightness distribution. The residual images are analyzed to ensure that there are no visible systematic deviations.

We have fitted 285 clusters from the initial sample with circular King (King 1962) and EFF (Elson et al. 1987) models. Since these models have different sensitivity to the cluster's "core" and "wings," comparison of the halflight radii derived by their means provides the robustness of the model fit and $r_{h}$. Elliptical models were not used in this study due to stochastic distribution of bright cluster stars, which introduce fake ellipticity for the lowmass clusters (see Deveikis et al. 2008 for a discussion of stochastic effects). A number of contaminating stars used for the model fitting was: 0 in 40\%, 1 in 30\%, and 2 in $20 \%$ cases. The maximum number of fitted stars was 6.

Typical fitted values of structural parameters are: (1) the core radius, $r_{c} \sim 0.75 \mathrm{pc}$, the concentration parameter, $c \sim 1.5$ (the King model); (2) the effective radius, $r_{e} \sim 0.75 \mathrm{pc}$, and the power-law index, $n \sim 1.3$ (the EFF model). For $\sim 70 \%$ of clusters the derived $c$ and $n$ parameter values were confined to the reasonable limits of $0.5 \leq c \leq 2.5$ (see boundaries for MW GCs in Harris 1996) and $1.05 \leq n \leq 3.5$, respectively. For practical convenience, the remaining $\sim 30 \%$ of clusters, having de- termined parameters outside these limits, were re-fitted by setting particular parameters to the corresponding boundary values. We note however, that although this slightly affects derived $r_{c}$ or $r_{e}$, it does not change the derived half-light radii nor introduces systematic deviations in the residual images.

Finally, intrinsic half-light radii of objects, based on the King, $r_{h}^{\mathrm{K}}$, and the EFF, $r_{h}^{\mathrm{E}}$, models were derived. They are plotted in Figure 1(a) and show a good correlation. The average half-light radii values, $r_{h}=\left(r_{h}^{\mathrm{K}}+\right.$ $\left.r_{h}^{\mathrm{E}}\right) / 2$, for 238 star clusters are provided in Table 1 .

To estimate both the robustness of the model fitting and the uncertainty of the derived half-light radius, $\sigma_{r_{h}}$, we have performed two tests: (1) varied photometric background value, $\mu_{\text {sky }}$, which is kept constant during model fitting; and (2) decreased fitting radius, $r_{\text {fit }}$, from the adopted initial value. These tests indicate that $r_{h}$ based on the King and the EFF models are in a good agreement, and the overall accuracy of the $r_{h}$ value is better than $20 \%$. The typical $\sigma_{r_{h}}$ is indicated by errorbars in Figure 1(a). Based on these results, we applied a conservative lower limit (which is mainly a subject to the resolution of ground-based observations) of $r_{h}=0.15^{\prime \prime}(0.6 \mathrm{pc})$ for the sample selection. Note however, that some clusters below this limit have been identified in the HST images, which turned out to be of a very compact nature or dominated by a central bright star.

To test the influence of PSF variability on the derived half-light radii, we have divided survey field into 15 regions and constructed individual PSFs for these regions. These PSF models were treated as "virtual objects" and analyzed as star clusters in attempt to derive their structural parameters. The derived half-light radii of eight "virtual objects" are displayed with asterisks in Figure 1(a) and are confined in the range of $r_{h}<0.4 \mathrm{pc}$. Note that only eight of 15 "virtual objects" are visible, since others failed to be fitted due to FWHM smaller than that of the adopted mosaic PSF. The scatter of $r_{h}<0.4$ pc corresponds to the $r_{h}$ uncertainty of $20 \%$ for objects with $r_{h} \sim 2 \mathrm{pc}$. It is the upper limit of uncertainty arising due to PSF variability across the survey field. For some individual clusters, we have additionally derived structural parameters by using local and mosaic PSFs, and found results being in a good agreement.

In case of semiresolved star clusters, subtraction of contaminating stars has a strong effect on the accuracy of derived half-light radii. We have automatically included all stars within $r_{\text {fit }}+$ FWHM $M_{\mathrm{PSF}}$ from the cluster's center and having magnitude $\leq\left(m_{\text {cluster }}+3\right)$ mag, i.e., up to 3 mag fainter than the cluster itself, into the fitting procedure. Also some individual strong contaminants were marked by hand. Conventional tests of varying the background level and the fitting radius indicate that $\sim 20 \%$ accuracy of $r_{h}$ is achieved. Visual inspection of residual images proved that clusters and contaminating stars are subtracted well. We have estimated the goodness of model fitting by comparing the standard deviation of the sky background within the cluster's area after model subtraction and that of the nearby sky region. For $\sim 90 \%$ of cases, these values were equal, except for the cases with larger residuals visible in the cluster's core, which, most probably, arise due to semiresolved stars. They are the 


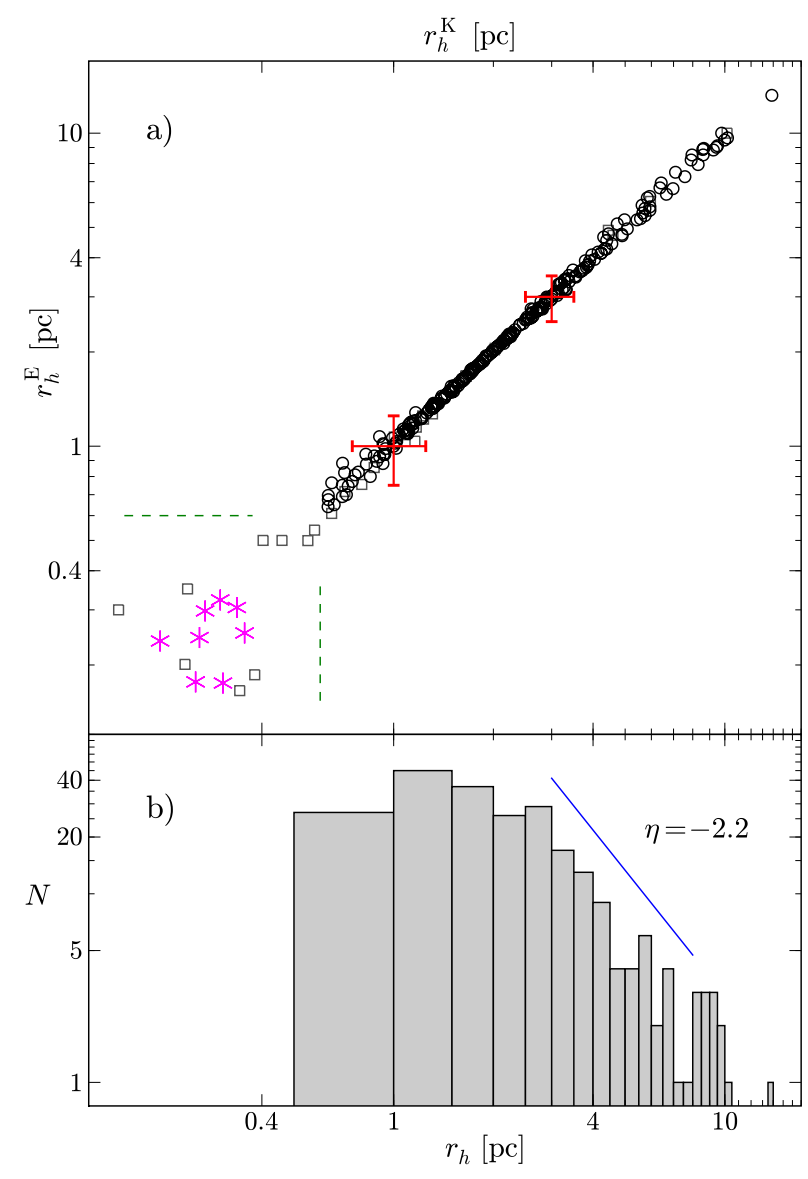

FIG. 1. - Half-light radii of star cluster candidates. (a) Determined half-light radii of 285 initial sample objects-based on the EFF model, $r_{h}^{\mathrm{E}}$, and the King model, $r_{h}^{\mathrm{K}}$ (squares mark rejected cluster candidates; circles - selected 238 clusters; asterisksPSFs constructed in the survey field and analyzed as "virtual objects;" dashed lines - the applied half-light radii selection limit $r_{h} \gtrsim 0.6 \mathrm{pc}$; error-bars - typical $\sigma_{r_{h}}$ ). (b) Histogram of average half-light radii, $r_{h}=\left(r_{h}^{\mathrm{K}}+r_{h}^{\mathrm{E}}\right) / 2$, of 238 clusters, overplotted with a slope $\eta=-2.2$ line, defined as $N\left(r_{h}\right) \mathrm{d} r_{h} \propto r_{h}^{\eta} \mathrm{d} r_{h}$.

main source of $r_{h}$ uncertainty for low-mass young star clusters (see Figure 3(c) in Deveikis et al. 2008).

We have compared $r_{h}$ values of 50 bright objects from our sample with the ones derived by Sablevičiūtè et al. (2007). Despite different analysis methods employed, we have found a good agreement between these two studies. The maximum $r_{h}$ difference of $\sim 30 \%$ is attributed to the influence of contaminating stars not properly accounted for in the previous study.

\subsection{Evolutionary Parameters}

The $U B V R I$ aperture CCD photometry data of 285 star clusters from the initial sample (Narbutis et al. 2008) were compared with SSP models (PÉGASE code: Fioc \& Rocca-Volmerange 1997) assuming the standard initial mass function (IMF; Kroupa 2002) and various metallicities to yield the best fit model evolutionary parameters, i.e., simple SSP model fitting was performed. The interstellar extinction was accounted for assuming the standard extinction law (Cardelli et al. 1989). The adopted parameter quantification technique and intrinsic precision analysis, based on the $U B V R I$ integrated photometry, was presented in detail by Narbutis et al.
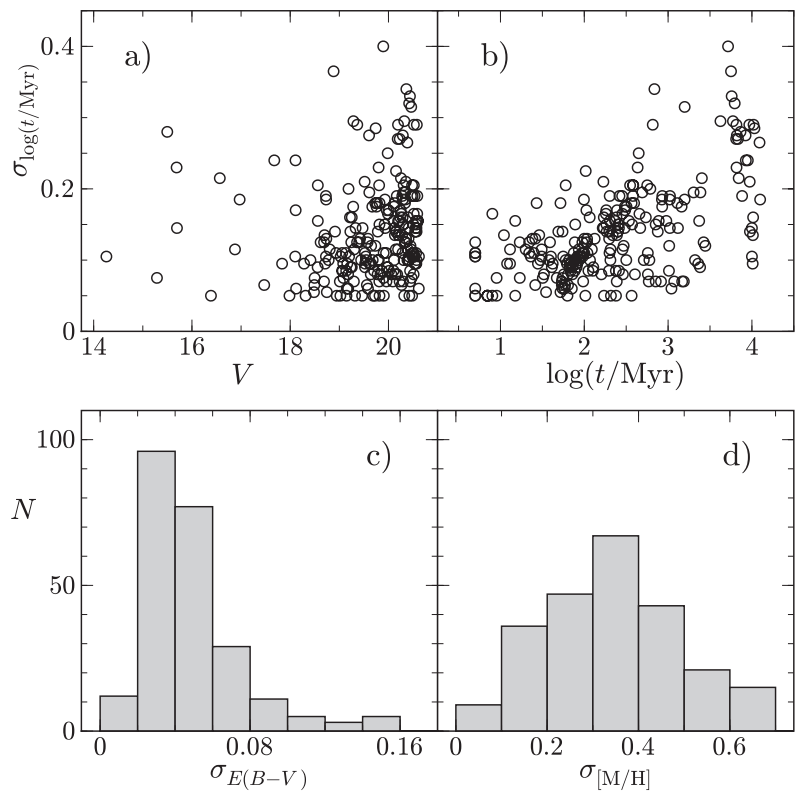

Fig. 2.- Accuracy of the derived evolutionary parameters of 238 star clusters. Panels (a) and (b) show the standard deviation of age, $\sigma_{\log (t / \mathrm{Myr})}$, plotted vs. $V$-band magnitude and vs. age, $\log (t / \mathrm{Myr})$, respectively. Panels (c) and (d) display the histograms of standard deviations: color excess, $\sigma_{E(B-V)}$, and metallicity, $\sigma_{[\mathrm{M} / \mathrm{H}]}$, respectively.

(2007a) and Bridžius et al. (2008). This technique, supplemented with a detailed investigation of multiband images, introduced to reduce the age-metallicity-extinction degeneracy, was used in the study.

The simple SSP model fitting results for 238 star clusters are provided in Table 1 . The determined evolutionary parameters are: absolute $V$-band magnitude (corrected for aperture correction, see below for details), $M_{V}$; age in Myr, $\log (t / \mathrm{Myr})$; mass in solar-mass units, $\log \left(m / m_{\odot}\right)$; metallicity, $[\mathrm{M} / \mathrm{H}]$; and color excess, $E(B-V)$.

The standard deviation of age, $\sigma_{\log (t / \mathrm{Myr})}$, is plotted versus $V$-band magnitude and age, $\log (t / \mathrm{Myr})$, in Figures 2(a) \& (b), respectively. The standard deviation of age increases typically from $\sim 0.1$ dex for young to $\sim 0.3$ dex for old objects. We note that systematic differences of the derived parameters could be expected if another SSP model bank would be used. The histograms of the standard deviation of color excess, $\sigma_{E(B-V)}$, and metallicity, $\sigma_{[\mathrm{M} / \mathrm{H}]}$, are shown in Figures $2(\mathrm{c}) \&(\mathrm{~d})$, respectively. The characteristic $\sigma_{E(B-V)}$ is $\sim 0.05$, reaching $\sim 0.15$ for $t \sim 10 \mathrm{Gyr}$ objects due to stronger ageextinction degeneracy. The typical $\sigma_{[\mathrm{M} / \mathrm{H}]}$ is $\sim 0.3$ dex, reaching up to $\sim 0.7$ dex for $t \sim 200$ Myr objects due to stronger age--metallicity degeneracy (see Narbutis et al. 2007a for parameter degeneracy maps).

We note that in this study numerous SSP model simplifications were assumed: (1) a constant IMF; (2) a standard for MW and constant for all cluster stars extinction law; (3) solar element ratio. Additionally, in cases of unreliable photometric solutions for metallicity, we restricted $[\mathrm{M} / \mathrm{H}]$ determination within lower and upper boundaries as a function of age taken from the chemical evolution model prediction. Referring to models of MW and M31 by Renda et al. (2005), we assumed that 


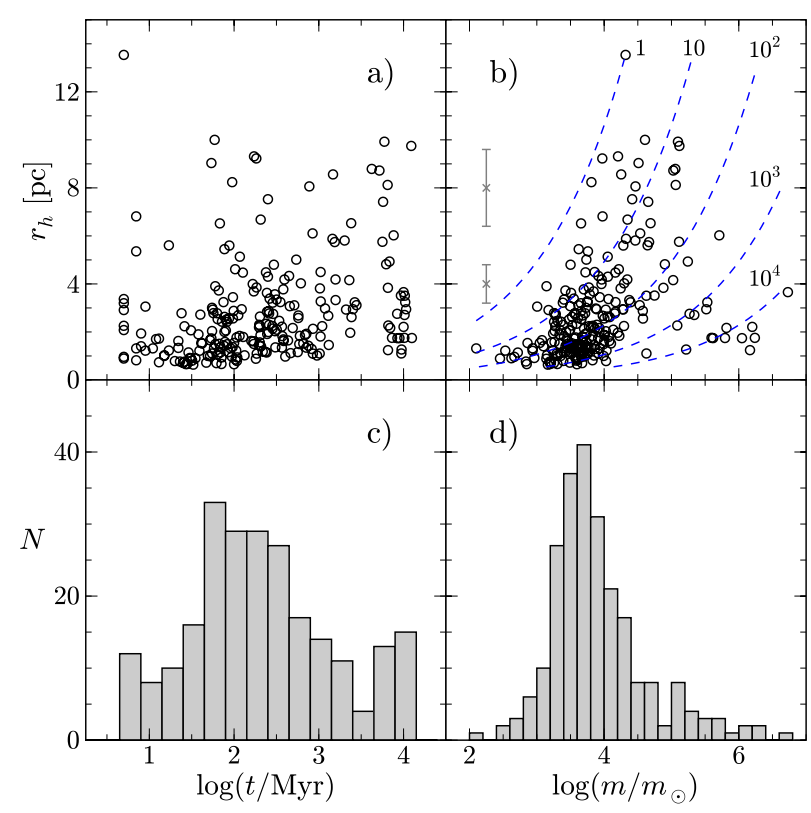

FIG. 3.- Parameters of 238 star clusters. (a) Half-light radius, $r_{h}$, plotted vs. age, $\log (t / \mathrm{Myr})$. (b) $r_{h}$ vs. $\operatorname{mass}, \log \left(m / m_{\odot}\right)$, overplotted with lines of a constant half-mass density, $\rho_{h}=1,10$ $10^{2}, 10^{3}$, and $10^{4} \mathrm{~m}_{\odot} \mathrm{pc}^{-3}$, from left to right, respectively. (c) and (d) Histograms of the derived cluster age, $\log (t / \mathrm{Myr})$, and mass, $\log \left(m / m_{\odot}\right)$, respectively. In panel (b) error-bars indicate typical $\sigma_{r_{h}}$.

metallicity evolution in the M31 survey field is not significantly different from that of solar neighborhood, which has been modeled by Schönrich \& Binnev (2009). Therefore, the $[\mathrm{M} / \mathrm{H}]$ boundaries roughly between -1 and +0.5 were considered, also taking into account possibility that oldest objects could be globular clusters in M31 halo with $[\mathrm{M} / \mathrm{H}]$ as low as -2 .

The mass of clusters in the solar-mass unit, $m / m_{\odot}$, was calculated equating the mass-to-luminosity ratio of the best fit SSP model of age, $t$, and metallicity, $[\mathrm{M} / \mathrm{H}]$, to the absolute $V$-band magnitude, $M_{V}$. The mass of clusters strongly depends on the derived age, therefore, the primary sources of mass uncertainty are the accuracy of age and extinction. Since cluster candidates in the crowded field were measured through small apertures, we have taken into account an individual aperture correction for each star cluster by applying the following procedure. Artificial clusters based on the best-fitted King model parameters were generated and convolved with the homogenized PSF of FWHM $=1.5^{\prime \prime}$; i.e., resolution of model cluster images was matched to the LGGS frame resolution employed for cluster photometry. Individual aperture used for a real cluster was applied to measure corresponding model cluster, the difference between measured and total flux was assumed as an aperture correction for a real cluster photometry. A typical $V$-band aperture correction is of $\sim 0.4 \mathrm{mag}$ and translates into a correction of mass by $\sim 0.15$ dex in $\log \left(m / m_{\odot}\right)$ scale.

Analysis of star cluster population requires a proper assessment of selection effects and resulting completeness. Stochastic modeling of clusters (Deveikis et al. 2008) unfolded a broad variety of their possible appearances, indicating that visual selection of low-mass clusters could be biased. However, for older clusters, i.e., after the phase of supergiants $(t \gtrsim 100 \mathrm{Myr})$, this problem appears to become less crucial.

\section{RESULTS AND DISCUSSION}

Here, we analyze the structural and evolutionary parameters of 238 star clusters in the southwestern field of the M31: half-light radius; age; mass; color excess; metallicity; and spatial distribution. Prior to presenting our results, we stress that important sources of systematic bias and uncertainty of the derived parameters, in particular for young low-mass star clusters, are stochastic effects (Deveikis et al. 2008). Assuming stochastic nature of star formation to randomly populate the stellar IMF, we modeled influence of stochastic effects on measurable cluster characteristics, and found that structural and evolutionary parameters could be biased - for some cluster age and mass intervals - in a systematic way. Recently, Barker et al. (2008) gave a strong caution that the standard SSP model analysis significantly underestimates uncertainty in the derived cluster age. However, only standard SSP model fitting was performed in this study; see a recent attempt to solve this problem for star clusters of solar metallicity by Maíz Apellániz (2009).

\subsection{Half-Light Radius}

The histogram of clusters' half-light radius, $r_{h}$, displayed in Figure 1(b), spans the range from $\sim 0.6 \mathrm{pc}$ to $\sim 10 \mathrm{pc}$ with one large object of $r_{h} \sim 14 \mathrm{pc}$ (KW249), which is described in the Appendix; the distribution peaks at $r_{h} \sim 1.5 \mathrm{pc}$. For comparison, the peak of the half-light radius distribution of MW GCs from Harris (1996, February 2003 rev. $^{5}$ ) catalog is at $\sim 2.5$ pc. Therefore, the majority of clusters from our M31 sample are smaller (more compact) than typical MW GCs.

The turnover and a lack of small-size clusters close to the applied lower limit of $r_{h}=0.6 \mathrm{pc}$ can be attributed to the visual selection of the cluster sample. The exponential decrease in the number of clusters in the larger halflight radii domain should be real. Note however, tests performed with "SimClust" (Deveikis et al. 2008) show that only extended clusters of low luminosity $\left(M_{V} \sim\right.$ -4.5) projected on star-forming regions could be missed by visual inspection. The overplotted power-law slope $\eta=-2.2$ line, defined as $N\left(r_{h}\right) \mathrm{d} r_{h} \propto r_{h}^{\eta} \mathrm{d} r_{h}$, was found as the best fit for M51 star clusters by Bastian et al. (2005), and agrees well with our star cluster size distribution in the range of $r_{h}=3-8 \mathrm{pc}$. However, a considerably steeper slope was found in the M51 cluster sample of Scheepmaker et al. (2007), which is five times more numerous than that of Bastian et al. (2005).

The half-light radius of clusters is plotted versus age, $\log (t / \mathrm{Myr})$, in Figure 3(a). No obvious half-light radius evolution with the age of cluster population, as has been found in LMC/SMC by Mackey \& Gilmore (2003), can be judged from this diagram, since objects with large $r_{h}$ are observed over a wide range of age. However, the lack of small-size clusters at age $t \sim 3 \mathrm{Gyr}$ can be attributed to the selection effects discussed by Hunter et al. (2003) for the LMC/SMC case, such that with increasing age only bright and, therefore, massive extended clusters are selected in the magnitude limited sample. At $t \sim 10 \mathrm{Gyr}$, halo GCs likely dominate in our sample and show $r_{h}$ distribution similar to that of the MW GCs population

\footnotetext{
${ }^{5}$ See http://physwww.mcmaster.ca/ harris/mwgc.dat
} 
with $r_{h}$ as small as $\sim 1$ pc. They have high densities (see Figure 3(b)) and likely have survived tidal disturbances by the disk.

The half-light radius versus mass, $\log \left(m / m_{\odot}\right)$, distribution is shown in Figure 3(b), overplotted with five lines of constant half-mass density, $\rho_{h}=3 m / 8 \pi r_{h}^{3}$, ranging from 1 to $10^{4} m_{\odot} \mathrm{pc}^{-3}$. The most numerous clusters in the M31 southwestern field are compact clusters of small size $r_{h} \sim 1.5 \mathrm{pc}$, intermediate mass $m \sim 4000 m_{\odot}$, and of young $t \sim 100$ Myr age. Largest clusters seem to be enveloped by the lines of $\rho_{h}$ from 1 to $100 m_{\odot} \mathrm{pc}^{-3}$, lending an impression that massive clusters tend to be bigger in size as far as clusters of relatively low specific density of $\rho_{h}<100 m_{\odot} \mathrm{pc}^{-3}$ are concerned (Hunter et al. 2003).

Comparing the $r_{h}$ versus mass distribution of our sample with a sample of M51 star clusters (Scheepmaker et al. 2007), we note a lack of objects with $4 \lesssim \log \left(m / m_{\odot}\right) \lesssim 5$ and $r_{h} \lesssim 5$ pc in our sample. The most massive, $\log \left(m / m_{\odot}\right) \gtrsim 4.5$, clusters are "branching" in Figure $3(\mathrm{~b})$ at small size, $r_{h} \lesssim 3 \mathrm{pc}$, and high density, $\rho_{h} \sim 10^{4} m_{\odot} \mathrm{pc}^{-3}$, which is characteristic to old GCs.

The oldest clusters, which have highest densities, are most likely halo GCs of small $r_{h}$. That the typical lifetime of $10^{4} m_{\odot}$ mass star cluster is estimated to be of $\sim 300$ Myr in the survey field (see Section 4.7 for details) implies very low probability for disk star clusters to reach an age of $\sim 10 \mathrm{Gyr}$ without disruption. However, radial velocity measurements are necessary to confirm whether these objects belong to halo.

\subsection{Age}

The distribution of clusters in the mass versus age diagram is shown in Figure 4 . The upper right domain of the diagram, $t \gtrsim 3$ Gyr and $4.0 \lesssim \log \left(m / m_{\odot}\right) \lesssim 7.0$, is occupied by $\sim 30$ classical GC candidates. The remaining $\sim 210$ are most likely disk clusters, which reside in the domain of $t \lesssim 3$ Gyr and $\log \left(m / m_{\odot}\right) \lesssim 4.5$. The curve along the lower envelope of cluster distribution is due to the selection limit of $V \lesssim 20.5 \mathrm{mag}$, which was calculated with PÉGASE SSP models of $[\mathrm{M} / \mathrm{H}]=-0.4$ dex metallicity, assuming zero extinction. Note, that star clusters are located above the selection limit due to aperture corrections taken into account for mass calculation. However, the upper envelope of the distribution should be free of selection effects.

Cerviño \& Luridiana (2004) have shown that SSP models cannot be used in a straightforward deterministic way for small-mass cluster analysis, when integrated luminosity of a model is lower than that of the brightest star included in the used isochrone, i.e., "Lowest Luminosity Limit" (LLL) requirement. They defined the smallest mass, $m^{\mathrm{LLL}}$, associated with the LLL, and the mass, $m^{\sigma \mathrm{LL}}$, corresponding to the $10 \%$ stochastic fluctuation of the $V$-band luminosity, $\sigma_{L_{V}}=0.1 \times L_{V}$, which translates into $\sim 0.04$ dex uncertainty of $\log \left(m / m_{\odot}\right)$. We have overplotted both parameters as a function of age in Figure 4, taken from Cerviño \& Luridiana (2004), noting that $m^{\sigma \mathrm{LL}}(t) \sim 10 \times m^{\mathrm{LLL}}(t)$. Note however, that SSP models of solar metallicity used by Cerviño \& Luridiana (2004) do not significantly influence a qualitative assessment of mass versus age distribution.

Judging from Figure $4, m^{\mathrm{LLL}}$ is $\log \left(m / m_{\odot}\right) \sim 4$ for

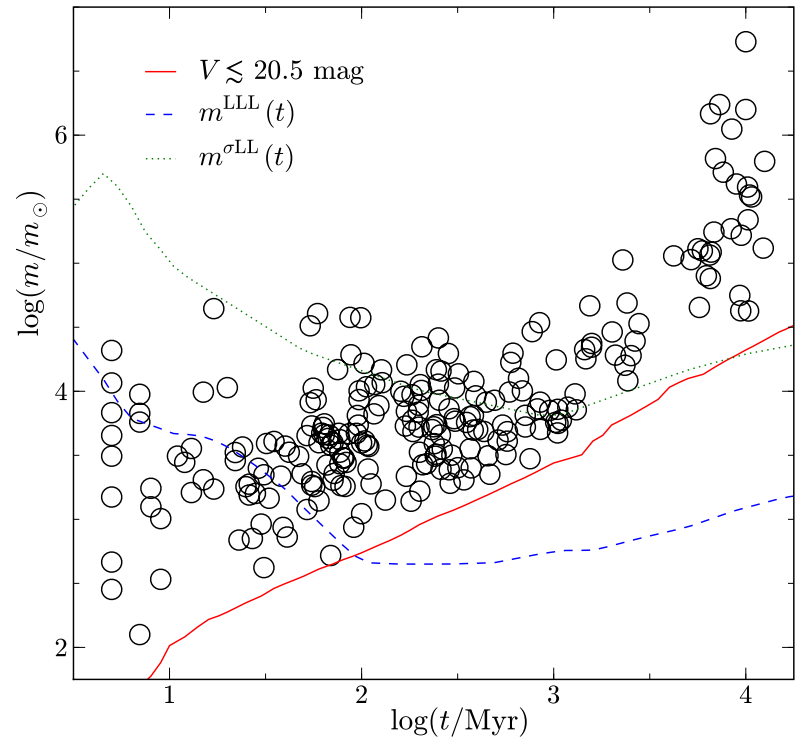

FIG. 4.- Mass vs. age diagram of 238 star clusters, overplotted with sample selection limit, $V \lesssim 20.5 \mathrm{mag}$, (solid line), the Lowest Luminosity Limit, $m^{\mathrm{LLL}}(t)$, (dashed line), and $10 \%$ stochastic fluctuation of cluster $V$-band luminosity, $m^{\sigma \mathrm{LL}}(t)$, (dotted line).

clusters younger than $\sim 10 \mathrm{Myr}$. At the age of $\sim 50 \mathrm{Myr}$, $m^{\text {LLL }}$ drops to $\log \left(m / m_{\odot}\right) \sim 3$, therefore, the majority of clusters from our sample satisfy the LLL requirement. At $t \sim 100 \mathrm{Myr}$, the $m^{\mathrm{LLL}}$ coincides with $V \lesssim 20.5 \mathrm{mag}$ selection limit; older objects are well above LLL. Therefore, clusters with $\log \left(m / m_{\odot}\right) \gtrsim 4.5$ should have the most accurate evolutionary parameters.

The stochastic effects on the derived cluster mass can be estimated by referring to the dotted line in Figure 4, based on the Cerviño \& Luridiana (2004) methods. The line displays $10 \%$ uncertainty of cluster's luminosity at a specific mass for a given age. Since we derived the mass from the $V$-band luminosity, the mass as a function of age, having $10 \%$ uncertainty of mass, is depicted by the dotted line in Figure 4. Formally, mass uncertainty increases to $100 \%$ at the LLL indicated by the dashed line. However, the uncertainty of mass is additionally affected by the uncertainty of the derived age, which can be evaluated from the slope of solid line in Figure 4, and also by the uncertainty of extinction shown in Figure 2. Therefore, the LLL provides only the lower limit for uncertainty arising due to stochastic effects, which primarily bias age and extinction, and consequently - mass, i.e., actual accuracy of evolutionary parameters is lower than it is shown in Figure 2.

The histogram of cluster age, displayed in Figure 3(c), spans the range from $t \sim 5 \mathrm{Myr}$ to $\sim 12 \mathrm{Gyr}$. Since evolutionary parameters of the clusters with $H \alpha$ emission are less reliable due to emission lines altering photometry and stochastic effects, we cannot properly estimate parameters of the clusters younger than $\sim 20 \mathrm{Myr}$. Therefore, we did not specifically target our survey for such star clusters.

The age distribution of clusters is displayed in Figure 5(a). We have calculated the number of clusters per linear age interval, $\mathrm{d} N / \mathrm{d} t$, by binning data in $\mathrm{d} \log (t / \mathrm{Myr})=0.2,0.3$, and 0.5 age intervals, while also shifting them by a half-bin width. These results were averaged and smoothed. The symbol size indicates an 

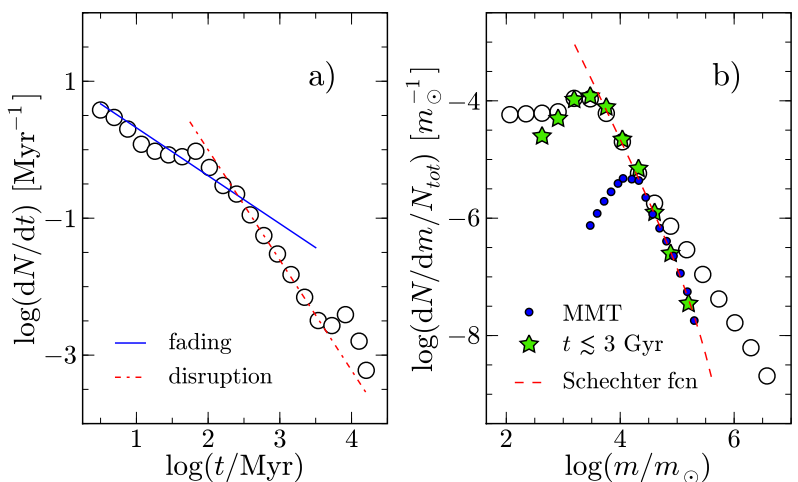

Fig. 5.- Age and mass distributions of 238 star clusters, indicated by open circles (size indicates the uncertainty of data binning) in both panels. (a) Number of clusters per age interval, $\log (\mathrm{d} N / \mathrm{d} t)$, vs. age, $\log (t / \mathrm{Myr})$ (circles), overplotted with power-law slopes expected for cluster population forming continuously and: (1) fading evolutionary below the detection limit (solid line); (2) instantaneously disrupting at some arbitrary time (dashdotted line). (b) Number of clusters per mass interval, normalized to the total number of clusters in the sample, $\log \left(\mathrm{d} N / \mathrm{d} m / N_{\text {tot }}\right)$, vs. mass, $\log \left(m / m_{\odot}\right)$ (circles), overplotted with Schechter's function (dashed line; Gieles 2009), adopting the turnover mass of $m^{*}=2 \times 10^{5} m_{\odot}$. Stars correspond to the intermediate age star clusters from our sample in-between of $\sim 100 \mathrm{Myr}$ and $\sim 3 \mathrm{Gyr}$; filled circles correspond to the star clusters from MMT spectroscopic survey by Caldwell et al. (2009, subsample $V<19.0 \mathrm{mag}$, ages in-between of $\sim 100 \mathrm{Myr}$ and an upper age limit of their sample $\sim 1 \mathrm{Gyr}$ ).

uncertainty of the resulting distribution, which is plotted versus age in a log scale. The age distribution is reasonably well represented with two power-law lines of different slopes, and is to be interpreted as a result of star cluster formation/disruption processes (see Section 4.7 for discussion).

The cluster number distribution $\mathrm{d} N / \mathrm{d} t$ (Figure $5(\mathrm{a})$ ) in general follows the "evolutionary fading" line. However, after subtracting the "evolutionary fading" number gradient, a gradual increase in the number of clusters per $\log$ age interval from $t \sim 20 \mathrm{Myr}$ to $t \sim 100 \mathrm{Myr}$ is obvious. A peak of the cluster number distribution is followed with a decrease in the number of clusters, which is a consequence of an evolutionary cluster fading effect, being always significant in the magnitude limited sample (see Figure 4), and star cluster disruption. We note that analysis of both Figures 3(c) \& 5(a) points to the peak of the cluster age distribution at $t \sim 70 \mathrm{Myr}$, suggesting an enhanced cluster formation episode at that epoch. A smaller secondary peak at $\sim 10$ Gyr is due to old GC candidates.

\subsection{Mass}

The histogram of cluster mass, displayed in Figure $3(\mathrm{~d})$, spans the range from $\sim 10^{2}$ to $\sim 5 \times 10^{6} \mathrm{~m}_{\odot}$ and shows a prominent peak at $m \sim 4000 m_{\odot}$. The decrease in the number of clusters in the low-mass domain is apparently not physical, but arises due to selection effects. The estimated completeness of our cluster sample is of $\sim 50 \%$ at $V \sim 20.5 \mathrm{mag}$ (Narbutis et al. 2008). Judging from these circumstances, the completeness at the age of $\sim 100$ Myr and the mass of $\sim 4000 m_{\odot}$ (Figure 4 ) should be credible, even taking into account a stochastic scattering of cluster luminosity at this age and mass. Therefore, decline in the number of clusters per log mass interval in the high-mass domain should be real.
The mass distribution of clusters is displayed in Figure 5(b). The number of clusters per linear mass interval, normalized to the total number of $N_{\text {tot }}=238$ clusters in our sample, $\mathrm{d} N / \mathrm{d} m / N_{\text {tot }}$, was calculated by binning data in $\mathrm{d} \log \left(m / m_{\odot}\right)=0.2,0.3$, and 0.5 mass intervals, and also shifting them by a half-bin width. These results were averaged and smoothed. The symbol size indicates the uncertainty of the resulting distribution. For a more detailed comparison of intermediate age (from $~ 100 \mathrm{Myr}$ to $\sim 3$ Gyr) star clusters from our sample we have used the selected subsample $(V<19.0 \mathrm{mag}$, age from $\sim 100 \mathrm{Myr}$ to an upper age limit of their sample $\sim 1 \mathrm{Gyr}$ ) of star clusters from the M31 disk study by Caldwell et al. (2009). The $\mathrm{d} N / \mathrm{d} m$ distribution presented in Caldwell et al. (2009) subsample was multiplied by 0.15 , taking into account that our survey field covers only $\sim 15 \%$ of the M31 disk. We note, that there are only eight objects in common between our sample and that of Caldwell et al. (2009).

Decline in the distribution of mass (Caldwell et al. 2009) less than $\log \left(m / m_{\odot}\right) \sim 4.3$ (see Figure $5(\mathrm{~b})$ ) arises due to a sample incompleteness and resembles a shape of our intermediate age sample distribution for mass less than $\log \left(m / m_{\odot}\right) \sim 3.5$. Although we have only few star clusters in the high-mass range, the good match with a $\mathrm{d} N / \mathrm{d} m$ slope (Caldwell et al. 2009) and extension down to the mass as $\operatorname{low}$ as $\log \left(m / m_{\odot}\right) \sim 3.7$ allows us to constrain a shape of the star cluster mass function for age in-between of $\sim 100 \mathrm{Myr}$ and $\sim 1$ Gyr. We overplotted Schechter's mass function from Gieles (2009, Equation (1)), adopting the turnover mass of $\mathrm{m}^{*}=2 \times 10^{5} \mathrm{~m}_{\odot}$, which shows a good agreement with both our intermediate age sample and Caldwell et al. (2009) subsample data. We note that only vertical adjustment for the Schechter's function was applied to match data at $m \sim 10^{4} m_{\odot}$.

Although we lack low-mass star clusters in our sample due to the selection limit at $V \sim 20.5 \mathrm{mag}\left(M_{V} \sim-4.0\right)$, the detailed HST study of star clusters in M31 by Krienke \& Hodge (2007, 2008) has revealed a numerous population of star cluster candidates as faint as $V \sim 23 \mathrm{mag}\left(M_{V} \sim-1.5\right)$. They are spatially well correlated with brighter counterparts of $t \sim 100 \mathrm{Myr}$ from our sample, therefore, it is reasonable to assume that they could be of a similar age. Thus, their mass should be significantly smaller than $\sim 4000 m_{\odot}$, corresponding to the mass range of typical MW OCs in the solar neighborhood (Piskunov et al. 2008). Although the parameters of MW star clusters are derived basing on individual stars, note that masses of MW clusters are a subject to the accuracy of their derived tidal radii, as discussed by Piskunov et al. (2008).

The high-mass domain of clusters from our sample is found to be overlapping well with the sample of $\sim 140$ young clusters, sharing the M31 disk rotation, studied using MMT spectra and HST images by Caldwell et al. (2009), who found a mass peak at $\log \left(m / m_{\odot}\right) \sim 4$, significantly higher than for our cluster sample, probably, due to brighter cluster selection limit applied for spectroscopy. The mass range of clusters from our sample younger than $\sim 3$ Gyr falls in-between of the MW GCs (McLaughlin \& van der Marel 2005) and the MW OCs (Piskunov et al. 2008), indicating that we are mainly dealing with the disk clusters from the intermediate mass 
range. The mass distribution of clusters older than $\sim 3$ Gyr overlaps well with the mass distribution of MW GCs (McLaughlin \& van der Marel 2005).

Comparing the mass versus age diagram of our cluster sample (Figure 4) with those of LMC/SMC (Hunter et al. 2003) and M51 (Bastian et al. 2005), we see a prominent lack of massive, $\log \left(\mathrm{m} / \mathrm{m}_{\odot}\right) \sim 5$, clusters younger than $\sim 3$ Gyr in M31. Although our survey covers only $\sim 15 \%$ of the deprojected M31 disk, it contains a representative part of its star-forming ring (Gordon et al. 2006) in the vicinity of NGC 206. We also note that other studies of the M31 disk clusters, which covered the whole galaxy, did not detect a significant number of clusters more massive than $\log \left(\mathrm{m} / \mathrm{m}_{\odot}\right) \sim 4.5$ in this age range either (Caldwell et al. 2009, and references therein).

A few massive, $\log \left(m / m_{\odot}\right) \sim 5$, objects with an estimated age of $t<1$ Gyr were reported by Caldwell et al. (2009), who found that most of their young clusters have a low concentration, typical to the MW OCs. Their spatial distribution is subject to patchy field selection of HST high-resolution imaging over the M31 disk. Although there are only eight objects in common in their and our samples, judging from the overlapping mass range and the reasonable agreement of the derived age, we may tentatively assume that both samples belong to the same category of star clusters.

The general lack of star clusters of $\log \left(m / m_{\odot}\right) \sim 5$ mass in the M31 disk could be due to a low-average star formation rate in M31 (Barmby et al. 2006). However, an unfavorable inclination angle of M31 could hide star clusters embedded in the M31 disk, mimicking the situation in MW (Clark et al. 2005) - there could be massive star clusters hidden by dust clouds and yet to be detected in M31. This hypothesis might be supported by the molecular cloud mass distribution in M31 (Nieten et al. 2006; Rosolowsky 2007), where star clusters with a mass of up to $\log \left(m / m_{\odot}\right) \sim 5$ are expected to form, if the typical star formation efficiency of $\sim 30 \%$ in the cores of molecular clouds is assumed.

\subsection{Extinction}

A typical color excess of star clusters in our sample is $E(B-V) \sim 0.25$. Clusters younger than $\sim 500 \mathrm{Myr}$ have a range of $E(B-V) \lesssim 1.0$, which is consistent with a large extinction of objects residing in the galaxy disk, e.g., four high extinction, $E(B-V) \sim 1.1$, objects of $t \sim 100 \mathrm{Myr}$ are projecting close to the $24 \mu \mathrm{m}$ emission regions and prominent dust lanes. The range of $E(B-V) \lesssim 0.4$ derived for clusters of intermediate age, $500 \mathrm{Myr} \lesssim t \lesssim 3 \mathrm{Gyr}$, might be related to the evolutionary fading of clusters and the observed narrow mass interval in this age range (see Figure 4), therefore, objects possessing color excess higher than $\sim 0.4$ are missing from our sample. We note that $E(B-V)$ versus projected distance from the M31 center does not show any tight correlation. Moreover, $E(B-V)$ of an individual cluster seems to be more strongly dependent on the local interstellar matter environment than on the radial distance from the M31 center.

The GC candidates of $t \gtrsim 3$ Gyr have color excess over the range of $E(B-V) \lesssim 0.6$, consistent with their presence in the M31 halo on both sides of the disk. There are numerous objects with $E(B-V) \sim 0.04$, which is in a good agreement with MW color excess in the direc-

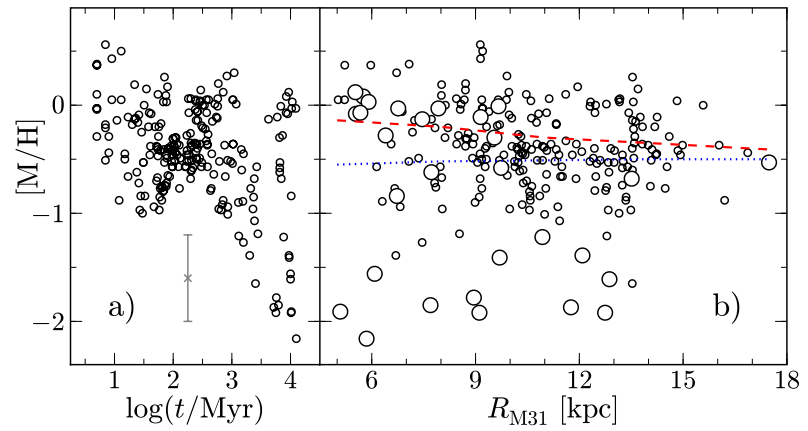

Fig. 6.- Metallicity, $[\mathrm{M} / \mathrm{H}]$, of 238 star clusters plotted vs. age, $\log (t / \mathrm{Myr})$, and projected distance from the M31 center, $R_{\mathrm{M} 31}$, in panels (a) and (b), respectively. In panel (a) error-bar indicates typical uncertainty of metallicity taken from Figure 2(d). In panel (b) clusters are divided into two age groups of $t \leqslant 3$ Gyr (small circles) and $t \gtrsim 3 \mathrm{Gyr}$ (large circles), and overplotted with the average metallicity trends for disk red giant stars from Worthey et al. (2005, dashed line) and Bellazzini et al. (2003, dotted line).

tion of the survey field in M31, estimated from the MW extinction maps (Schlegel et al. 1998).

Note however, that for eight objects in common with Caldwell et al. (2009) sample, which have $E(B-V) \sim$ 0.25 derived in their study, we determine color excess from 0.05 to 0.65 . Although Caldwell et al. (2009) determined extinction by examining the shape of spectra continuum, for those cases when late-type stars dominate they used a mean color excess for young clusters of $E(B-V) \sim 0.28$, which is in agreement with the typical value of $E(B-V) \sim 0.25$ derived in our study for presumably the same population of star clusters.

\subsection{Metallicity}

The metallicity, $[\mathrm{M} / \mathrm{H}]$, of star clusters, plotted versus age and projected distance from the M31 center, $R_{\mathrm{M} 31}$, in Figures $6(\mathrm{a}) \&(\mathrm{~b})$, respectively, spans a range from -2.0 to +0.5 dex. We divided clusters into two age groups of $t \lesssim 3 \mathrm{Gyr}$ and $t \gtrsim 3 \mathrm{Gyr}$, indicated by a different symbol size in Figure $6(\mathrm{~b})$. The apparent narrowing of the metallicity scatter at an age of $\sim 200 \mathrm{Myr}$ is attributed to the SSP model fitting artifact due to a strong age--metallicity degeneracy in this age domain (Narbutis et al. 2007a). Although the accuracy of photometrically derived metallicity is low (see Figure 2(d)), the evolutionary trend with time is apparent.

A rather constant average metallicity of $[\mathrm{M} / \mathrm{H}] \sim$ -0.4 dex is observed for clusters younger than $\sim 1 \mathrm{Gyr}$, however, there is a slight tendency of the average $[\mathrm{M} / \mathrm{H}]$ to decrease with increasing galactocentric distance. The radial profile of cluster metallicity matches well those of disk red giant stars from Worthey et al. (2005) and Bellazzini et al. (2003) shown by lines in Figure 6(b).

There are 20 clusters in our sample, which have [Fe/H] estimates from the William Herschel Telescope spectra by Perrett et al. (2002). By accounting for $\alpha$-element overabundance typical to MW GCs, we see a reasonable agreement between photometric and spectroscopic metallicity estimates of old, $t \sim 10 \mathrm{Gyr}$, clusters. Two of those metal-rich massive clusters (KW221 and KW225), presumably similar to GCs, have metallicity of $[\mathrm{M} / \mathrm{H}] \sim$ -0.8 and $0.0 \mathrm{dex}$, and are found in our survey field area closest to the M31 bulge, located at the projected distance of $R_{\mathrm{M} 31} \sim 5 \mathrm{kpc}$ from the galaxy's center. Note however, that for several objects of $\sim 100 \mathrm{Myr}$ age, 


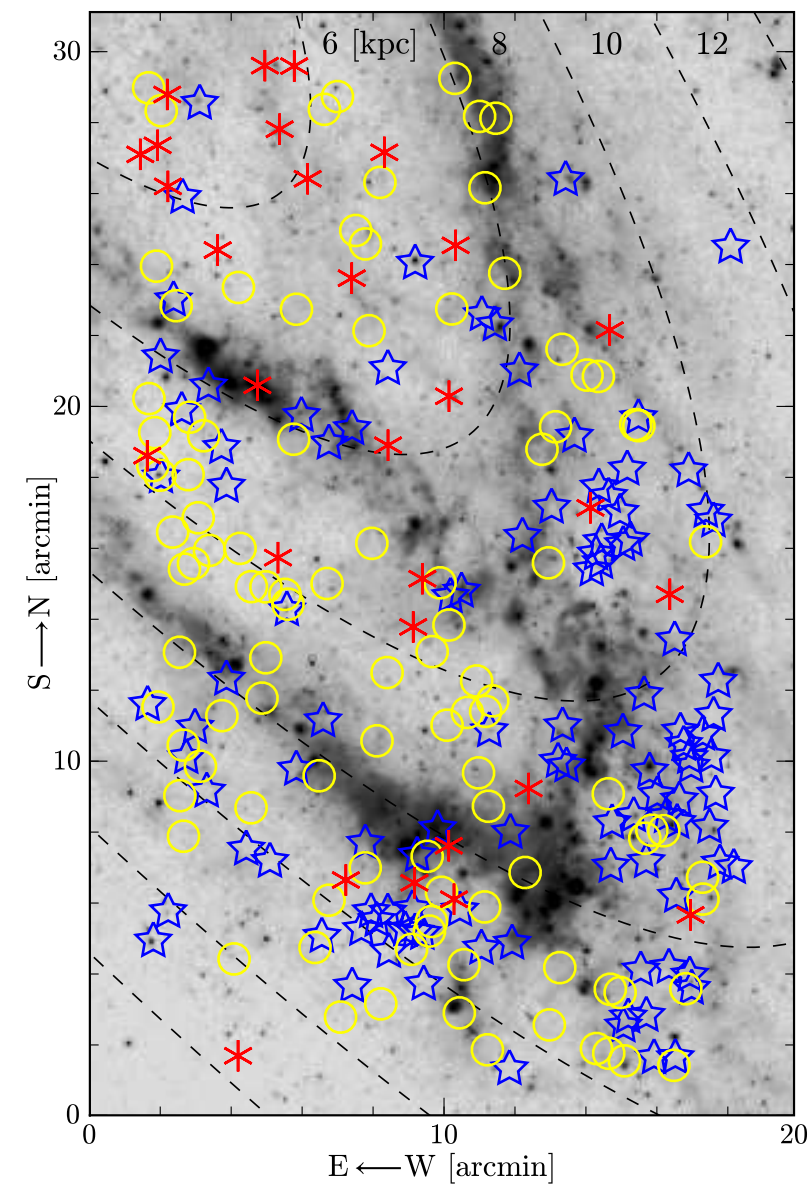

FIG. 7.- Distribution of 238 star clusters in the M31 SW field, overlaid on Spitzer $(24 \mu \mathrm{m})$ image. Clusters are divided into three age groups: $t \lesssim 150 \mathrm{Myr}$ (stars), $150 \mathrm{Myr} \lesssim t \lesssim 3 \mathrm{Gyr}$ (circles), and $t \gtrsim 3$ Gyr (asterisks). Elliptical ring segments, indicating projected distance from the M31 center, $R_{\mathrm{M} 31}=6-18 \mathrm{kpc}$, are marked with dashed lines. North is up, east is left.

the metallicity estimated by Perrett et al. (2002) is of $[\mathrm{Fe} / \mathrm{H}] \sim-2.2$ dex, i.e., significantly lower than a photometrically estimated $[\mathrm{M} / \mathrm{H}] \sim-0.4$ dex.

The elaborated M31 evolution models, such as "M31 model-b" by Renda et al. (2005), may well reproduce the metallicity gradient and the metallicity change in time, as they are presented in Figure 6. Detailed studies of the age-dependent profiles of the disk cluster population would provide further constraints on the M31 disk evolution models.

\subsection{Spatial Distribution}

Positions of star clusters are indicated on the Spitzer $(24 \mu \mathrm{m})$ image of the M31 southwestern field in Figure 7 . Clusters are divided into three age groups: young $(t \lesssim 150 \mathrm{Myr}) ;$ intermediate $(150 \mathrm{Myr} \lesssim t \lesssim 3 \mathrm{Gyr}) ;$ and old $(t \gtrsim 3 \mathrm{Gyr})$, shown by different symbols. The distribution of young clusters resembles that of warm dust, but is shifted outward to larger $R_{\mathrm{M} 31}$ by $\sim 1.5 \mathrm{kpc}$ along the galaxy's major axis. The intermediate age clusters are smoothly distributed over the whole field. Old age clusters (GC candidates) scatter over the area, but show a higher concentration toward the M31 bulge.

Star clusters with ultraviolet $(G A L E X)$ or $24 \mu \mathrm{m}$ (Spitzer) emission are mainly distributed along the sites of active star formation. At the bottom of the survey field, young and intermediate age clusters appear to be well mixed. Around the position of $\left[6^{\prime}, 16^{\prime}\right]$, where a lack of warm dust is attributed to the "split" of the star forming ring, intermediate age clusters dominate. However, around $\left[17^{\prime}, 11^{\prime}\right]$ mainly young clusters are observed. The spatial density of young and intermediate age clusters decreases toward the bulge, which is consistent with a gas distribution in the M31 disk (Nieten et al. 2006).

To explain the M31 spiral structure and features of the prominent star-forming ring at $R_{\mathrm{M} 31} \sim 10 \mathrm{kpc}$, two scenarios of the M32 galaxy having passed through the M31 disk were recently proposed: (1) passage trough the disk occurred $\sim 20$ Myr ago (Gordon et al. 2006) and created a "split" of the ring around position $\left[6^{\prime}, 16^{\prime}\right]$ (see Figure 7; NGC 206 is located at the ring's "branching" point $\left.\left[15^{\prime}, 17^{\prime}\right]\right)$; (2) passage trough the M31 center occurred $\sim 210$ Myr ago (Block et al. 2006). The star cluster population data could provide a reference to disentangle the more preferable scenario.

True locations of objects in the M31 disk are subject to a strong projection effect due to an unfavorable inclination angle of $\sim 75^{\circ}$. The projection of the galaxy and its rotation are such that the southwestern part of the disk approaches the observer (Henderson 1979); the corotation radius lies beyond $R_{\mathrm{M} 31} \sim 20 \mathrm{kpc}$ (Efremov 1980; Ivanov 1985); and the rotation period at $R_{\mathrm{M} 31} \sim 10 \mathrm{kpc}$ distance is of $\sim 250 \mathrm{Myr}$. Assuming that all clusters in our sample are residing in the M31 disk, we made a deprojection of cluster locations to face-on view, and referring to the disk rotation curve (Carignan et al. 2006), computed object positions as a function of look-back time.

Judging by eye, the clumps of young clusters, which are located at $\left[9^{\prime}, 5^{\prime}\right]$ and $\left[17^{\prime}, 10^{\prime}\right]$, formed two compact spatial configurations $\sim 80 \pm 20 \mathrm{Myr}$ ago. This age coincides well with the cluster age peak (see Section 4.2) and suggests a possible evolutionary connection. There was no other compact spatial cluster configuration noted in the look-back time range from $\sim 20 \mathrm{Myr}$ to $\sim 150 \mathrm{Myr}$, which is valid for this kind of study based on our cluster sample. The range of the age distribution peak, estimated from the SSP model fitting, is of $\sim 50-120 \mathrm{Myr}$. However, there is an age-metallicity degeneracy observed at the age of $\sim 100 \mathrm{Myr}$, which could slightly bias the age determination. Therefore, we note this interesting coincidence of cluster age peak and compact spatial cluster configurations. To discuss this finding more reliably, a global kinematic study of the M31 disk cluster population and a detailed dynamic model of the M31 and M32 encounter are needed. Note however, that Caldwell et al. (2009) did not find evidence for a peak in star formation between 20 Myr and 1 Gyr.

\subsection{Cluster Formation and Disruption}

If star clusters are being formed continuously at a constant rate with a power-law cluster initial mass function, their number increases with time in the mass versus age diagram as described by Boutloukos \& Lamers (2003). Consequently, the mass of the most massive cluster in the older log age bins rises due to a statistical effect of sample size. However, in our sample, we see a lack of massive $\log \left(m / m_{\odot}\right) \gtrsim 4$ clusters older than $100 \mathrm{Myr}$ (Figure 4), probably, indicating a nonconstant cluster formation history. Therefore, using the 
observed mass and age distributions of star clusters we attempt to derive their formation and disruption rates in the studied field by adopting the technique developed by Boutloukos \& Lamers (2003).

For a simple estimate, first we selected clusters more massive than $\log \left(\mathrm{m} / \mathrm{m}_{\odot}\right) \sim 3.5$. There are $N_{1} \sim 30$ and $N_{2} \sim 70$ objects in the age ranges $\Delta t_{1} \equiv 30 \lesssim$ $t \lesssim 100 \mathrm{Myr}$ and $\Delta t_{2} \equiv 100 \mathrm{Myr} \lesssim t \lesssim 1 \mathrm{Gyr}$, respectively. If a cluster formation rate ( $\widetilde{C F R})$ is constant and there is no cluster disruption, then a ratio of $N_{2} / N_{1}=\Delta t_{2} / \Delta t_{1} \approx 13$ is expected. The actual ratio is $N_{2} / N_{1} \sim 2$, indicating that CFR is not constant and/or significant cluster disruption took place during the last Gyr in the M31 disk.

The number of clusters per linear age interval, $\mathrm{d} N / \mathrm{d} t$, plotted versus age in Figure 5(a) can be described by two power-law slopes, intersecting at $t \sim 300 \mathrm{Myr}$. The slope values were taken from the fading/disruption models by Boutloukos \& Lamers (2003, Equation (14): $\alpha=2.0$, $\gamma=0.62, \zeta=0.69)$ and only vertical adjustment to the observed distribution was applied. In the age domain of $t \lesssim 300$ Myr the slope line, expected for a cluster population forming continuously and fading in the course of evolution below the detection limit, provides a rather good match to the observed data. Around $t \sim 70 \mathrm{Myr}$, there is a sudden increase in the number of clusters by a factor of $\sim 2$, which could be attributed to the increased CFR at that epoch. In the age domain older than $~ 300 \mathrm{Myr}$, the observed distribution can be well described by the slope expected for a cluster disruption at some arbitrary time. An increase in the cluster number at $t \sim 10 \mathrm{Gyr}$ is attributed to the GC candidates, prominent in our sample.

The number of clusters per linear mass interval, $\mathrm{d} N / \mathrm{d} m$, is displayed versus mass in Figure 5(b). In the mass range of $\log \left(m / m_{\odot}\right) \lesssim 3.3$ the incompleteness of our cluster sample is obvious. Due to incompleteness, the mass distribution of our cluster sample cannot be used to constrain the typical lifetime of star clusters since the fading line cannot be adjusted properly. Note that the distribution of clusters in between $\sim 100$ Myr and $\sim 3 \mathrm{Gyr}$ and in the mass range of $\log \left(m / m_{\odot}\right) \gtrsim 4.5$ is steeper than that of the whole sample, which includes the old GC candidates.

Using the Boutloukos \& Lamers (2003) technique and relying on the power-law slope line intersection point in Figure 5(a), we estimate a conservative value of typical lifetime of $\log \left(\mathrm{m} / \mathrm{m}_{\odot}\right)=4$ mass cluster to be of $t_{4}^{\text {dis }} \sim 300$ Myr in the M31 survey field. For comparison, the following values in other galaxies were derived by Lamers et al. (2005): M51 central region- 70 Myr; M33 — 630 Myr; MW solar neighborhood- 560 Myr; and $\mathrm{SMC}-\sim 8 \mathrm{Gyr}$.

The cluster disruption analysis gives the disruption time of $\sim 500 \mathrm{Myr}$ if only cluster age distribution of our sample is used. If the mass distribution is taken into account (although it is difficult to define intersection point of fading and disruption lines in Figure 5(b)), the cluster disruption time can be estimated to be as small as $\sim 100$ Myr since it is sensitive to the "intersection" mass. Therefore, we choose $t_{4}^{\text {dis }} \sim 300 \mathrm{Myr}$ as a conservative value. We note that a similar inconsistency between age and mass distribution analysis was noticed recently for LMC star clusters by Parmentier \& de Grijs (2008). However, they found an opposite effect for the LMC sample - the cluster mass distribution analysis tends to yield higher cluster disruption timescale than the age distribution analysis. Such discrepancy might be related to the assumptions applied in the Boutloukos \& Lamers (2003) analysis used in our study: (1) a constant cluster formation rate; (2) a power-law cluster initial mass function, which should be of the Schechter's function type as shown in Section 4.3 .

Lamers et al. (2005) found anticorrelation between the lifetime, $t_{4}^{\text {dis }}$, of $\log \left(\mathrm{m} / \mathrm{m}_{\odot}\right)=4$ mass cluster and the ambient density of its environment, $\rho_{\mathrm{amb}}$, in the galaxies. Following the reasoning by Lamers et al. (2005), we attempt to estimate $\rho_{\mathrm{amb}}$ in the M31 disk. We took the structural parameters of the M31 disk from the "best-fit model" of Geehan et al. (2006): a disk scale-length, $R_{d}=5.4 \mathrm{kpc}$; a central surface density, $\Sigma_{0}=4.6 \times 10^{8} m_{\odot} \mathrm{kpc}^{-2}$. Therefore, the average disk surface density at $R_{\mathrm{M} 31} \sim 10 \mathrm{kpc}$, i.e., at the center of our survey field $\Sigma=\Sigma_{0} \exp \left(-R_{\mathrm{M} 31} / R_{d}\right) \sim 70 m_{\odot} \mathrm{pc}^{-2}$.

van der Kruit (2002) has shown that for spiral galaxies the vertical scale height is of $h_{z} \sim 0.15 R_{d}$; this implies $h_{z}^{\text {stars }} \sim 800$ pc. Braun (1991) found $h_{z}^{\text {gas }} \sim 350$ pc for a gas distribution at a galactocentric distance of $R_{\mathrm{M} 31}=$ $10 \mathrm{kpc}$. The scale height for dust distribution is assumed to be of $h_{z}^{\text {dust }} \sim 100$ and $\sim 150$ pc by Hatano et al. (1997) and Semionov et al. (2003), respectively. Depending on the adopted $h_{z}$, the estimated ambient density in our survey field, $\rho_{\mathrm{amb}} \sim 0.5 \Sigma h_{z}^{-1}$, is in the range of $\rho_{\mathrm{amb}} \sim$ $0.05 \ldots 0.35 m_{\odot} \mathrm{pc}^{-3}$. This density is much lower than the typical half-mass density $\rho_{h} \sim 10^{2} m_{\odot} \mathrm{pc}^{-3}$ of star clusters, shown in Figure 3(b).

Referring to Figure 4 in Lamers et al. (2005), we see that values of $t_{4}^{\text {dis }} \sim 300 \mathrm{Myr}$ and $\rho_{\text {amb }} \sim$ $0.05 \ldots 0.35 m_{\odot} \mathrm{pc}^{-3}$ derived here, place the studied field of the M31 disk in the position between MW and M33 and M51, however, slightly below the predicted theoretical $t_{4}^{\text {dis }}=t_{4}^{\text {dis }}\left(\rho_{\mathrm{amb}}\right)$ line. Gieles et al. (2006) have modeled encounters of star clusters with molecular clouds to explain short disruption time for star clusters in the M51 central region. Our survey field in M31 is centered on the star-forming ring possessing high-density gas (Nieten et al. 2006), therefore, enhanced star cluster disruption in respect to MW and M33 could apparently take place. Although Rosolowsky (2007, and references therein) discuss similarity of the molecular cloud properties in M31 and MW, enhanced star cluster disruption should apparently take place in the M31 molecular gas "ring" in respect to that of the solar neighborhood. The whole field survey of the M31 disk clusters and more detailed models of cluster and cloud kinematics, which are needed to estimate encounter cross sections in the disk, should be considered in future.

Finally, we attempt to estimate the CFR in the M31 disk. We select $N=51$ clusters with age $\Delta t \equiv 30 \mathrm{Myr} \lesssim$ $t \lesssim 130$ Myr and mass $3.3 \lesssim \log \left(\mathrm{m} / \mathrm{m}_{\odot}\right) \lesssim 4.5$ (see Figure 4). This subsample should have accurately estimated parameters and be relatively free of selection and cluster disruption effects. The total stellar mass of these clusters is $\Delta m \sim 3 \times 10^{5} m_{\odot}$. Assuming the constant power-law cluster mass function (index - 2.0; Gieles 2009) down to $\log \left(m / m_{\odot}\right)=2$, mass correction factor of $\sim 2.5$ was de- 
duced. Therefore, the approximate rate of star formation in the clusters was $\mathrm{CFR}_{\text {field }}=\Delta m / \Delta t \sim 0.008 m_{\odot} \mathrm{yr}^{-1}$. The deprojected area of survey field (Figure 7 ) is of $\sim 70 \mathrm{kpc}^{2}$, making $\sim 15 \%$ of the galaxy disk. From this, we estimate the lower limit of the average CFR at the epoch of $\sim 100 \mathrm{Myr}$ to be of $\mathrm{CFR}_{\mathrm{M} 31} \sim 0.05 m_{\odot} \mathrm{yr}^{-1}$ over the M31 disk. This can be compared to the presentday star formation rate of $0.4 m_{\odot} \mathrm{yr}^{-1}$ (Barmby et al. 2006), indicating that $\gtrsim 10 \%$ of formed stars could remain "locked" in star clusters during 100 Myr.

For comparison, Williams (2003) found the mean star formation rate for the disk $\sim 1 m_{\odot} \mathrm{yr}^{-1}$ over the last 60 Myr. Based on stellar population analysis, he suggests that the lowest star formation rate occurred $\sim 25 \mathrm{Myr}$ ago being $\sim 2$ times lower compared with the epoch from $\sim 50 \mathrm{Myr}$ to $\sim 250 \mathrm{Myr}$. Note, a good coincidence of star formation history in M31 deduced by Williams (2003) and one inferred from our cluster population data (Figure 5(a)), which suggests an episode of a double increase in cluster formation $\sim 70 \mathrm{Myr}$ ago. Also, Kodaira et al. (1999) have found that the star formation rate in the field around OB association A24 has decreased by $\sim 2.5$ times from the epoch at $\sim 1$ Gyr to the present day.

Although clusters in the intermediate mass range $\left(3.5 \lesssim \log \left(m / m_{\odot}\right) \lesssim 4.5\right)$ are not well known in the MW, the compact clusters in M31 might represent a new class of star clusters of intermediate age $30 \mathrm{Myr} \lesssim t \lesssim 3 \mathrm{Gyr}$, linking the age of GC formation and the present. It could be suspected that this class of disk-population clusters is not observed in the MW because of high extinction through the Galactic plane. This might be supported by the Clark et al. (2005) discovery that a Galactic star cluster Westerlund 1 is a Super Star Cluster with a mass of up to $\log \left(m / m_{\odot}\right) \sim 5$, age of $\sim 3 \mathrm{Myr}$, and radius of $0.6 \mathrm{pc}$, which suffers strong interstellar extinction of $A_{V} \sim 11.5 \mathrm{mag}$ being at the distance of $\sim 5 \mathrm{kpc}$. Therefore, a more numerous massive compact star cluster population also could be hidden by dust clouds in the M31 disk.

\section{SUMMARY}

The sample of compact star clusters in the present homogeneous photometric survey of the $17.5^{\prime} \times 28.5^{\prime}$ field in the southwestern part of the M31 disk is apparently overlapping with the clusters detected in the patchy $H S T$ fields, in terms of their mass $3.0 \lesssim \log \left(m / m_{\odot}\right) \lesssim 4.5$, which is in-between of the mass of typical OCs and classical GCs, well known in the MW. The structural properties of the compact clusters are similar to those of GCs, except for minor irregularities, and they resemble those of massive clusters in the LMC/SMC, including blue clusters of globular appearance. The metallicity of sample clusters is rather constant over a wide range of galactocentric distances, with a typical value scattering around $[\mathrm{M} / \mathrm{H}] \sim-0.4$ dex.

The global follow-up survey of the whole M31 disk cluster population, based on photometry and size measurement, is of great interest and importance to comprehend the evolution of galaxy disks. Supplemented with spectroscopic and higher image resolution studies, as well as modeling of cluster stochastic effects, it would provide a guiding panoramic view and better constraints for the M31 and M32 interaction models.
We are grateful to the anonymous referee for constructive suggestions, which helped to improve the paper considerably. This work was financially supported in part by a Grant of the Lithuanian State Science and Studies Foundation. The star cluster survey is based on the Suprime-Cam images, collected at the Subaru Telescope, which is operated by the National Astronomical Observatory of Japan. The research is based in part on archival data obtained with the Spitzer Space Telescope, and has made use of the following: the NASA/IPAC Extragalactic Database (NED) and the NASA/IPAC Infrared Science Archive, which are operated by the Jet Propulsion Laboratory, California Institute of Technology, under contract with the National Aeronautics and Space Administration; the SAOImage DS9, developed by Smithsonian Astrophysical Observatory; the USNOFS Image and Catalog Archive operated by the United States Naval Observatory, Flagstaff Station. The data presented in this paper were partly obtained from the Multimission Archive at the Space Telescope Science Institute.

\section{APPENDIX}

\section{OBJECTS FOR DETAILED STUDY}

Here, we present several remarkable objects, which could serve as targets for future spectroscopy and highresolution imaging. We briefly discuss their properties referring to the derived parameters, multiband and $H S T$ images. We provide cross-referencing with The Revised Bologna Catalog of M31 Globular Clusters and candidates compiled by Galleti et al. (2007) in brackets when available.

KW044 (B325) is located at the position of $\left[16^{\prime}, 2^{\prime}\right]$ in Figure 7 and is resolved in Suprime-Cam images. The structural model fit resulted in a large half-light radius, $r_{h} \sim 11 \mathrm{pc}$, and an extremely small ratio of King model parameters, $r_{t} / r_{c} \sim 3$. Its age of $\sim 60 \mathrm{Myr}$, mass of $\log \left(m / m_{\odot}\right) \sim 4.6$, and radial velocity of $-560 \mathrm{~km} \mathrm{~s}^{-1}$, compatible with gas disk velocity at this position, imply that KW044 is a representative of the young disk cluster population. However, another solution for evolutionary parameters (a low extinction case) also existsthe cluster could be an old object of low concentration and low metallicity, resembling the extended ones in M31 (Mackey et al. 2006) and M33 (Stonkutè et al. 2008), and suggesting its location in the M31 halo. The age of $\sim 630 \mathrm{Myr}$ is derived by Caldwell et al. (2009).

$\mathrm{KW} 072(\mathrm{VdBO})$ is located at the position of $\left[15^{\prime}, 7^{\prime}\right]$ in Figure 7. A visual impression that it is surrounded by an enhanced surface number density of stars (ejected from cluster) is confirmed by the structural model fit, indicating its extended nature - structural model parameters: ratio $r_{t} / r_{c} \sim 300$ (King), $n \sim 1$ (EFF), and $r_{h} \sim 5.5 \mathrm{pc}$. Its young age of $\sim 20$ Myr is confirmed by a strong ultraviolet $(G A L E X)$ flux. A mass, $\log \left(m / m_{\odot}\right) \sim 4.6$, of the cluster, which formed recently, is remarkably high comparing with the MW typical OCs' mass. This cluster was studied in detail by Perina et al. (2009) based on photometry of individual stars from $H S T$ images. The parameters derived are in a very good agreement with values derived in the present study based on its integrated properties.

KW141 (B011D) is located at the position of $\left[11^{\prime}, 15^{\prime}\right]$ in Figure 7. It represents a population of young, $\sim 10$ Myr, intermediate-mass, $\log \left(\mathrm{m} / \mathrm{m}_{\odot}\right) \sim 3.8$, clusters. 


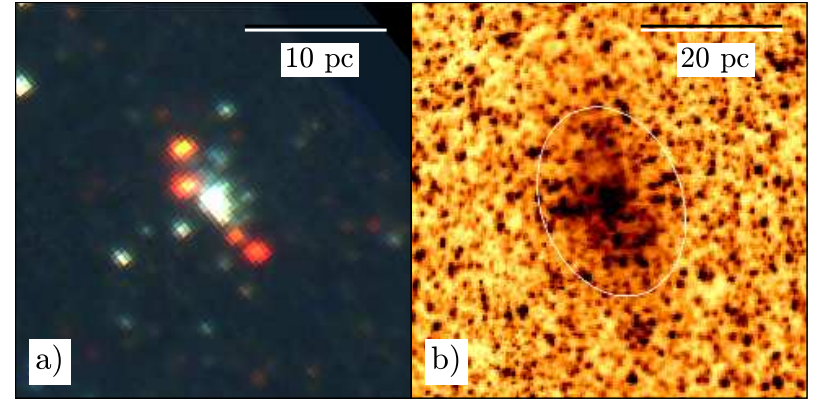

FIG. 8.- HST images. Panels show: (a) KW141 - an example of stochastic appearance of red bright stars (color coding of HST filters in the electronic edition: red-F814W, green-F555W, and blue-F439W); (b) KW249 - an object with the largest half-light radius, $r_{h} \sim 14 \mathrm{pc}$, in our sample (F606W filter, overplotted with ellipse indicating $r_{h}$, derived via elliptical King model fit). Note different image scales in panels (a) and (b). North is up, east is left.

However, stochastically appearing four bright red stars enclose the blueish central part of this object suggesting an older age. Its $H S T$ WFPC2 color image is displayed in Figure 8(a). The age and mass of this "stochastic cluster example" are in good agreement with Caldwell et al. (2009): $\sim 30$ Myr and $\log \left(m / m_{\odot}\right) \sim 3.8$.

KW249 - the most controversial object, located close to the M31 bulge at the position of $\left[3^{\prime}, 29^{\prime}\right]$ in Figure 7. Its young age of $\sim 10$ Myr and a mass of $\log \left(\mathrm{m} / \mathrm{m}_{\odot}\right) \sim 4.3$ make it incompatible with the structural parameter bestfit model indicating very high ratio of $r_{t} / r_{c}>500$ (King model) and large half-light radius of $r_{h} \sim 14 \mathrm{pc}$ (the most extended object in our cluster sample). It is associated with $24 \mu \mathrm{m}$ (Spitzer) and HI emission, probably, still being an embedded cluster with $E(B-V) \sim 1.5$, which could explain the absence of $H \alpha$ and ultraviolet emission. It was imaged with the HST WFPC2 (F606W filter), displayed in Figure 8(b) (fitted elliptical models provide the ellipticity of $\sim 0.75$ - a ratio of minor to major axis). We suggest, that it could be either: (1) an intermediate-mass cluster, which expanded after a rapid gas removal phase (Baumgardt \& Kroupa 2007), or (2) a background galaxy, having emission in $K_{\mathrm{s}}$ (2MASS) attributed to older stellar populations.
KW271 \& KW273 (G099*) — a double cluster candidate, located at the position of $\left[2^{\prime}, 18^{\prime}\right]$ in Figure 7 . KW271 is centered on a strong $24 \mu \mathrm{m}$ emission source, its parameters are: $t \sim 10 \mathrm{Myr}, \log \left(\mathrm{m} / \mathrm{m}_{\odot}\right) \sim 3.7$, $E(B-V) \sim 0.9$, and $r_{h} \sim 3.6$ pc. Contrarily, KW273 has no $24 \mu \mathrm{m}$ emission, is much older $(t \sim 800 \mathrm{Myr})$, more massive $\left(\log \left(m / m_{\odot}\right) \sim 4.5\right)$, has a low color excess $(E(B-V) \sim 0.1)$, and large half-light radius $\left(r_{h} \sim\right.$ $4.3 \mathrm{pc})$. The structural parameters of two "adhered" cluster candidates were derived by masking KW271 and fitting the brighter KW273. After subtracting the best-fit model of KW273 from the original image, KW271 was fitted. In total, seven iterations of the object subtraction were performed, leading to fine residual images. We suggest two possible explanations of the nature of these objects: (1) they make a double cluster, similar to those detected in LMC/SMC (Carvalho et al. 2008), with one component just emerging from the embedded phase. Close image inspection reveals an extended "halo" enshrouding both these objects, however, the derived age difference does not support this case; (2) both clusters (they could be a cluster and a galaxy $-24 \mu \mathrm{m}$ emission source) are subject to projection, however, probability of such close coincidence is low. We note, that Caldwell et al. (2009) classify KW271 as a background galaxy based on the measured redshift $z \sim 0.15$, and for KW273 they provide age of $\sim 400 \mathrm{Myr}$ and mass $\log \left(m / m_{\odot}\right) \sim 4.0$ in reasonable agreement with our values.

KW279 (B205D) is located at a large projected galactocentric distance, thus far from the star-forming ring, at the position of $\left[2^{\prime}, 5^{\prime}\right]$ in Figure 7 . It is associated with a strong $24 \mu \mathrm{m}$ emission, which supports its young age of $\sim 10$ Myr. A low-mass, $\log \left(m / m_{\odot}\right) \sim 3.2$, substantial color excess, $E(B-V) \sim 0.6$, and a moderate size, $r_{h} \sim 2 \mathrm{pc}$, were deduced assuming it being a star cluster. However, its asymmetric shape with a blueish "tail," pointing at a neighboring $24 \mu \mathrm{m}$ emission source, implies that it could be an interacting starburst galaxy. We note, that Caldwell et al. (2009) classify KW279 as a background galaxy based on the measured redshift $z \sim 0.1$.

\section{REFERENCES}

Barker, S., de Grijs, R., \& Cerviño, M. 2008, A\&A, 484, 711

Barmby, P., Holland, S., \& Huchra, J. P. 2002, AJ, 123, 1937

Barmby, P., MacLaughlin, D. E., Harris, W. E., Harris, G. L. H., \& Forbes, D. A. 2007, AJ, 133, 2764

Barmby, P., et al. 2006, ApJ, 650, L45

Bastian, N., Gieles, M., Lamers, H. J. G. L. M., Scheepmaker, R. A., \& de Grijs, R. 2005, A\&A, 431, 905

Baumgardt, H., \& Kroupa, P. 2007, MNRAS, 380, 1589

Bellazzini, M., Cacciari, C., Federici, L., Fusi Pecci, F., \& Rich, M. 2003, A\&A, 405, 867

Block, D. L., et al. 2006, Nature, 443, 832

Boutloukos, S. G., \& Lamers, H. J. G. L. M. 2003, MNRAS, 338, 717

Braun, R. 1991, ApJ, 372, 54

Bridžius, A., Narbutis, D., Stonkutè, R., Deveikis, V., \& Vansevičius, V. 2008, Balt. Astron., 17, 337

Caldwell, N., Harding, P., Morrison, H., Rose, J. A., Schiavon, R., \& Kriessler, J. 2009, AJ, 137, 94

Cardelli, J. A., Clayton, G. C., \& Mathis, J. S. 1989, ApJ, 345, 245

Carignan, C., Chemin, L., Huchtmeier, W. K., \& Lockman, F. J. 2006, ApJ, 641, L109
Carvalho, L., Saurin, T. A., Bica, E., Bonatto, C., \& Schmidt, A. A. 2008, A\&A, 485, 71

Cerviño, M., \& Luridiana, V. 2004, A\&A, 413, 145

Clark, J. S., Negueruela, I., Crowther, P. A., \& Goodwin, S. P. 2005, A\&A, 434, 949

Cohen, J. G., Matthews, K., \& Cameron, P. B. 2005, ApJ, 634, L45

de Vaucouleurs, G. 1958, ApJ, 128, 465

Deveikis, V., Narbutis, D., Stonkutè, R., Bridžius, A., \&

Vansevičius, V. 2008, Balt. Astron., 17, 351

Efremov, Y. N. 1980, Sov. Astron. Lett., 6, 152

Elson, R. A. W., Fall, S. M., \& Freeman, K. C. 1987, ApJ, 323, 54

Fioc, M., \& Rocca-Volmerange, B. 1997, A\&A, 326, 950

Galleti, S., Bellazzini, M., Federici, L., Buzzoni, A., \& Fusi Pecci, F. 2007, A\&A, 471, 127

Geehan, J. J., Fardal, M. A., Babul, A., \& Guhathakurta, P. 2006, MNRAS, 366, 996

Gieles, M. 2009, MNRAS, 394, 2113

Gieles, M., Portegies Zwart, S. F., Baumgardt, H., Athanassoula, E., Lamers, H. J. G. L. M., Sipior, M., \& Leenaarts, J. 2006, MNRAS, 371, 793

Gordon, K. D., et al. 2006, ApJ, 638, L87

Harris, W. E. 1996, AJ, 112, 1487 
Hatano, K., Branch, D., Fisher, A., \& Starrfield, S. 1997, ApJ, 487, L45

Henderson, A. P. 1979, A\&A, 75, 311

Hill, A., \& Zaritsky, D. 2006, AJ, 131, 414

Hunter, D. A., Elmegreen, B. G., Dupuy, T. J., \& Mortonson, M. 2003, AJ, 126, 1836

Ivanov, G. R. 1985, Ap\&SS, 110, 357

King, I. 1962, AJ, 67, 471

Kodaira, K. 2002, Rev. Mod. Astron., 15, 1

Kodaira, K., Vansevičius, V., Bridžius, A., Komiyama, Y., Miyazaki, S., Stonkutè, R., Šablevičiūtè I., \& Narbutis, D. 2004, PASJ, 56, 1025

Kodaira, K., Vansevičius, V., Stonkutè, R., Narbutis, D., \& Bridžius, A. 2008, in ASP Conf. Ser. 399, Panoramic Views of Galaxy Formation and Evolution, ed. T. Kodama, T. Yamada, \& K. Aoki (San Francisco, CA: ASP), 431

Kodaira, K., Vansevičius, V., Tamura, M., \& Miyazaki, S. 1999, ApJ, 519, 153

Krienke, O. K., \& Hodge, P. W. 2007, PASP, 119, 7

Krienke, O. K., \& Hodge, P. W. 2008, PASP, 120, 1

Kroupa, P. 2002, Science, 295, 82

Lamers, H. J. G. L. M., Gieles, M., \& Portegies Zwart, S. F. 2005, A\&A, 429, 173

Mackey, A. D., \& Gilmore, G. F. 2003, MNRAS, 338, 85

Mackey, A. D., et al. 2006, ApJ, 653, L105

Maíz Apellániz, J. 2009, ApJ, 699, 1938

Massey, P., Olsen, K. A. G., Hodge, P. W., Strong, S. B., Jacoby, G. H., Schlingman, W., \& Smith, R. C. 2006, AJ, 131, 2478

McConnachie, A. W., Irwin, M. J., Ferguson, A. M. N., Ibata,

R. A., Lewis, G. F., \& Tanvir, N. 2005, MNRAS, 356, 979

McLaughlin, D. E., \& van der Marel, R. P. 2005, ApJS, 161, 304

Miyazaki, S., et al. 2002, PASJ, 54, 833

Narbutis, D., Bridžius, A., Stonkutè, R., \& Vansevičius, V. 2007a, Balt. Astron., 16, 421

Narbutis, D., Stonkutè, R., \& Vansevičius, V. 2006, Balt. Astron., 15,471

Narbutis, D., Vansevičius, V., Kodaira, K., Bridžius, A., \& Stonkutè, R. 2007b, Balt. Astron., 16, 409

Narbutis, D., Vansevičius, V., Kodaira, K., Bridžius, A., \& Stonkute, R. 2008, ApJS, 177, 174

Narbutis, D., Vansevičius, V., Kodaira, K., Šablevičiūtè, I., Stonkute, R., \& Bridžius, A. 2006, Balt. Astron., 15, 461
Nieten, C., Neininger, N., Guélin, M., Ungerechts, H., Lucas, R., Berkhuijsen, E. M., Beck, R., \& Wielebinski, R. 2006, A\&A, 453, 459

Parmentier, G., \& de Grijs, R. 2008, MNRAS, 383, 1103

Perina, S., et al. 2009, A\&A, 494, 933

Perrett, K. M., Bridges, T. J., Hanes, D. A., Irwin, M. J., Brodie, J. P., Carter, D., Huchra, J. P., \& Watson, F. G. 2002, AJ, 123, 2490

Piskunov, A. E., Schilbach, E., Kharchenko, N. V., Röser, S., \& Scholz, R.-D. 2008, A\&A, 477, 165

Renda, A., Kawata, D., Fenner, Y., \& Gibson, B. K. 2005, MNRAS, 356, 1071

Rosolowsky, E. 2007, ApJ, 654, 240

Šablevičiūtè, I., Vansevičius V., Kodaira, K., Narbutis, D., Stonkutè, R., \& Bridžius, A. 2006, Balt. Astron., 15, 547

Šablevičiūtè, I., Vansevičius V., Kodaira, K., Narbutis, D., Stonkutè, R., \& Bridžius, A. 2007, Balt. Astron., 16, 397

Scheepmaker, R. A., Haas, M. R., Gieles, M., Bastian, N., Larsen, S. S., \& Lamers, H. J. G. L. M. 2007, A\&A, 469, 925

Schlegel, D. J., Finkbeiner, D. P., \& Davis, M. 1998, ApJ, 500, 525

Schönrich, R., \& Binney, J. 2009, MNRAS, 396, 203

Semionov, D., Stonkutè, R., \& Vansevičius, V. 2003, Balt. Astron., 12, 633

Stetson, P. B. 1987, PASP, 99, 191

Stonkutè, R., et al. 2008, AJ, 135, 1482

Tody, D. 1993, in ASP Conf. Ser. 52, Astronomical Data Analysis Software and Systems II, ed. R. J. Hanisch, R. J. V.

Brissenden, \& J. Barnes (San Francisco, CA: ASP), 173

van der Kruit, P. C. 2002, in ASP Conf. Proc., 273, The Dynamics, Structure \& History of Galaxies, ed. G. S. Da Costa \& H. Jerden (San Francisco, CA: ASP), 7

Williams, B. F. 2003, AJ, 126, 1312

Williams, B. F., \& Hodge, P. W. 2001a, ApJ, 548, 190

Williams, B. F., \& Hodge, P. W. 2001b, ApJ, 559, 851

Worthey, G., España, A., MacArthur, L. A., \& Courteau, S. 2005, ApJ, 631, 820

TABLE 1

Evolutionary and Structural Parameters of High-Probability Star Cluster Candidates in the Southwest Part of the M31 Disk

\begin{tabular}{ccccccccc}
\hline \hline ID & $\alpha_{\mathrm{J} 2000}$ & $\delta_{\mathrm{J} 2000}$ & $M_{V}{ }^{\mathrm{a}}$ & $\log (t / \mathrm{Myr})^{\mathrm{b}}$ & $\log \left(m / m_{\odot}\right)^{\mathrm{c}}$ & {$[\mathrm{M} / \mathrm{H}]^{\mathrm{d}}$} & $E(B-V)^{\mathrm{e}}$ & $r_{h}{ }^{\mathrm{f}}$ \\
\hline KW003 & 10.04519 & 40.89759 & -6.0 & 1.89 & 3.42 & -0.5 & 0.12 & 2.0 \\
KW004 & 10.04568 & 40.60326 & -6.6 & 1.67 & 3.48 & -0.9 & 0.10 & 0.7 \\
KW006 & 10.05430 & 40.60535 & -6.7 & 1.79 & 3.66 & -0.2 & 0.21 & 0.7 \\
KW007 & 10.05468 & 40.69164 & -6.1 & 2.04 & 3.56 & -0.4 & 0.52 & 0.9 \\
KW008 & 10.05671 & 40.63810 & -6.1 & 1.52 & 3.16 & -0.8 & 0.30 & 0.6 \\
KW009 & 10.05700 & 40.76773 & -5.2 & 1.36 & 2.79 & -0.1 & 0.34 & 0.8 \\
KW010 & 10.05768 & 40.67549 & -7.3 & 2.07 & 4.01 & -0.5 & 0.70 & 5.1 \\
KW011 & 10.05924 & 40.65584 & -6.6 & 1.88 & 3.66 & -0.8 & 0.16 & 1.4 \\
KW013 & 10.06067 & 40.62244 & -6.6 & 1.78 & 3.61 & -0.4 & 0.02 & 1.5 \\
KW014 & 10.06177 & 40.77234 & -6.3 & 1.42 & 3.26 & -0.1 & 0.50 & 2.2 \\
KW015 & 10.06203 & 40.75771 & -4.9 & 2.85 & 3.70 & 0.1 & 0.05 & 1.9 \\
KW017 & 10.06453 & 40.66658 & -6.9 & 1.72 & 3.64 & -0.7 & 0.22 & 1.0 \\
KW019 & 10.06504 & 40.58820 & -5.2 & 2.56 & 3.54 & -0.3 & 0.13 & 1.3 \\
KW020 & 10.06519 & 40.59857 & -5.1 & 3.39 & 4.07 & -1.1 & 0.09 & 2.6 \\
KW022 & 10.07194 & 40.65138 & -7.0 & 1.74 & 3.72 & -0.4 & 0.03 & 3.0 \\
KW023 & 10.07237 & 40.79158 & -6.8 & 1.60 & 3.55 & -0.5 & 0.41 & 2.7 \\
KW024 & 10.07249 & 40.54608 & -5.1 & 1.59 & 2.92 & -0.1 & 0.20 & 1.2 \\
KW025 & 10.07289 & 40.58077 & -7.0 & 3.71 & 5.02 & -1.9 & 0.52 & 8.7 \\
KW026 & 10.07313 & 40.65559 & -6.6 & 1.83 & 3.63 & -0.8 & 0.05 & 1.5 \\
KW027 & 10.07362 & 40.55231 & -6.1 & 1.89 & 3.51 & 0.0 & 0.25 & 2.6 \\
KW028 & 10.07611 & 40.54573 & -7.0 & 2.89 & 4.46 & -0.7 & 0.04 & 8.1 \\
KW029 & 10.07649 & 40.66179 & -5.0 & 1.61 & 2.85 & -0.4 & 0.02 & 1.5 \\
KW031 & 10.07849 & 40.66804 & -6.3 & 1.42 & 3.18 & -0.7 & 0.04 & 0.8 \\
KW032 & 10.07944 & 40.63540 & -6.6 & 1.62 & 3.51 & -0.1 & 0.39 & 1.5 \\
KW033 & 10.08092 & 40.62481 & -7.4 & 1.73 & 3.90 & -0.4 & 0.30 & 1.7 \\
KW034 & 10.08174 & 40.58977 & -5.7 & 1.74 & 3.25 & 0.1 & 0.32 & 2.0 \\
KW035 & 10.08174 & 40.71139 & -6.1 & 1.91 & 3.46 & -0.7 & 0.19 & 2.5 \\
KW037 & 10.08296 & 40.51322 & -6.3 & 2.00 & 3.59 & -0.5 & 0.07 & 0.7 \\
KW038 & 10.08386 & 40.50941 & -5.5 & 2.85 & 3.81 & -0.9 & 0.14 & 1.3 \\
\hline
\end{tabular}


TABLE 1 - Continued

\begin{tabular}{|c|c|c|c|c|c|c|c|c|}
\hline ID & $\alpha_{\mathrm{J} 2000}$ & $\delta_{\mathrm{J} 2000}$ & $M_{V}^{\mathrm{a}}$ & $\log (t / \mathrm{Myr})^{\mathrm{b}}$ & $\log \left(m / m_{\odot}\right)^{\mathrm{c}}$ & {$[\mathrm{M} / \mathrm{H}]^{\mathrm{d}}$} & $E(B-V)^{\mathrm{e}}$ & $r_{h}{ }^{\mathrm{f}}$ \\
\hline KW039 & 10.08462 & 40.73291 & -9.3 & 3.82 & 6.15 & -0.6 & 0.15 & 1.2 \\
\hline KW040 & 10.08659 & 40.55609 & -6.5 & 1.97 & 3.66 & -0.5 & 0.15 & 1.0 \\
\hline KW041 & 10.08782 & 40.62758 & -4.7 & 1.96 & 2.92 & -0.4 & 0.03 & 1.3 \\
\hline KW042 & 10.08929 & 40.62074 & -5.9 & 2.69 & 3.92 & -0.6 & 0.21 & 2.2 \\
\hline KW043 & 10.09325 & 40.63796 & -6.3 & 1.40 & 3.24 & -0.2 & 0.35 & 0.7 \\
\hline KW044 & 10.09623 & 40.51320 & -9.2 & 1.77 & 4.59 & -0.8 & 0.60 & 10.0 \\
\hline KW045 & 10.09674 & 40.62105 & -5.9 & 2.27 & 3.68 & -0.1 & 0.22 & 1.1 \\
\hline KW046 & 10.09793 & 40.64923 & -6.3 & 1.45 & 3.19 & -0.8 & 0.18 & 0.7 \\
\hline KW048 & 10.10042 & 40.60629 & -8.4 & 1.30 & 4.02 & -0.2 & 0.19 & 0.8 \\
\hline KW049 & 10.10088 & 40.53335 & -6.0 & 1.92 & 3.44 & -0.4 & 0.03 & 1.5 \\
\hline KW050 & 10.10110 & 40.68546 & -5.4 & 1.72 & 3.07 & -0.4 & 0.03 & 1.7 \\
\hline KW051 & 10.10121 & 40.61724 & -4.5 & 2.26 & 3.13 & 0.1 & 0.02 & 0.9 \\
\hline KW053 & 10.10341 & 40.81300 & -7.6 & 2.40 & 4.40 & -0.5 & 0.37 & 7.5 \\
\hline KW054 & 10.10363 & 40.81692 & -7.8 & 2.01 & 4.20 & -0.4 & 0.69 & 4.6 \\
\hline KW055 & 10.10418 & 40.55488 & -5.5 & 1.91 & 3.24 & -0.4 & 0.02 & 2.4 \\
\hline KW056 & 10.10534 & 40.81352 & -6.9 & 2.41 & 4.15 & -0.4 & 0.38 & 2.8 \\
\hline KW057 & 10.10801 & 40.62815 & -9.8 & 0.84 & 3.97 & -0.5 & 1.15 & 6.8 \\
\hline KW058 & 10.10906 & 40.75886 & -6.1 & 1.69 & 3.34 & -0.2 & 0.47 & 1.0 \\
\hline KW059 & 10.11086 & 40.79259 & -7.4 & 2.11 & 4.05 & -0.8 & 0.46 & 1.8 \\
\hline KW061 & 10.11223 & 40.53262 & -7.3 & 1.99 & 3.99 & -0.4 & 0.63 & 2.6 \\
\hline KW062 & 10.11367 & 40.75677 & -6.7 & 1.98 & 3.73 & -0.6 & 0.12 & 1.4 \\
\hline KW064 & 10.11449 & 40.66775 & -5.9 & 1.74 & 3.28 & -0.5 & 0.24 & 1.1 \\
\hline KW065 & 10.11469 & 40.51168 & -4.9 & 2.46 & 3.37 & 0.0 & 0.03 & 3.2 \\
\hline KW066 & 10.11525 & 40.52838 & -5.6 & 1.48 & 2.99 & -1.0 & 0.05 & 0.9 \\
\hline KW067 & 10.11554 & 40.77210 & -7.7 & 1.04 & 3.48 & -0.1 & 0.93 & 1.3 \\
\hline KW068 & 10.11716 & 40.54411 & -5.0 & 2.71 & 3.61 & 0.0 & 0.04 & 3.1 \\
\hline KW070 & 10.12135 & 40.85848 & -6.3 & 3.77 & 5.08 & -0.1 & 0.30 & 9.9 \\
\hline KW071 & 10.12160 & 40.62482 & -5.4 & 1.78 & 3.13 & -0.8 & 0.15 & 3.9 \\
\hline KW072 & 10.12255 & 40.60420 & -10.0 & 1.23 & 4.63 & -0.7 & 0.21 & 5.6 \\
\hline KW073 & 10.12259 & 40.77968 & -4.7 & 1.49 & 2.61 & -0.5 & 0.04 & 0.9 \\
\hline KW075 & 10.12328 & 40.54568 & -5.2 & 3.12 & 3.84 & -1.2 & 0.06 & 1.5 \\
\hline KW076 & 10.12387 & 40.63842 & -5.8 & 2.58 & 3.81 & -0.4 & 0.30 & 1.7 \\
\hline KW077 & 10.12448 & 40.51532 & -5.7 & 2.55 & 3.74 & -0.2 & 0.27 & 3.2 \\
\hline KW078 & 10.12708 & 40.75818 & -8.5 & 1.18 & 3.97 & -0.5 & 0.03 & 1.0 \\
\hline KW079 & 10.12764 & 40.74831 & -7.1 & 1.11 & 3.54 & 0.5 & 0.03 & 1.7 \\
\hline KW081 & 10.12861 & 40.83648 & -6.7 & 2.40 & 4.04 & -0.5 & 0.50 & 4.3 \\
\hline KW082 & 10.12872 & 40.78416 & -5.6 & 2.03 & 3.38 & -0.3 & 0.07 & 0.8 \\
\hline KW084 & 10.13069 & 40.75239 & -5.1 & 0.95 & 2.52 & 0.4 & 0.03 & 1.2 \\
\hline KW085 & 10.13216 & 40.51760 & -4.8 & 2.30 & 3.21 & -0.3 & 0.17 & 1.6 \\
\hline KW086 & 10.13340 & 40.74484 & -5.9 & 0.70 & 2.44 & -0.0 & 0.09 & 0.9 \\
\hline KW087 & 10.13404 & 40.77417 & -6.0 & 3.76 & 4.64 & -1.8 & 0.26 & 7.4 \\
\hline KW089 & 10.13572 & 40.83714 & -6.3 & 2.29 & 3.83 & -0.3 & 0.07 & 2.2 \\
\hline KW094 & 10.14379 & 40.80833 & -6.7 & 1.11 & 3.19 & 0.1 & 0.65 & 0.7 \\
\hline KW096 & 10.14847 & 40.93010 & -6.8 & 1.18 & 3.30 & -0.5 & 0.83 & 1.0 \\
\hline KW097 & 10.14988 & 40.65192 & -6.9 & 1.81 & 3.73 & -0.9 & 0.47 & 1.1 \\
\hline KW099 & 10.15130 & 40.84943 & -6.1 & 3.44 & 4.52 & -1.2 & 0.18 & 3.1 \\
\hline KW100 & 10.15220 & 40.67090 & -8.0 & 1.94 & 4.27 & -0.4 & 0.51 & 5.6 \\
\hline KW101 & 10.15475 & 40.55608 & -6.2 & 2.82 & 4.08 & -1.0 & 0.42 & 1.5 \\
\hline KW102 & 10.15519 & 40.65407 & -7.5 & 1.76 & 3.92 & -1.0 & 0.55 & 1.3 \\
\hline KW103 & 10.15562 & 40.81270 & -6.4 & 2.23 & 3.84 & -0.3 & 0.10 & 3.7 \\
\hline KW105 & 10.15837 & 40.77468 & -4.8 & 2.00 & 3.03 & -0.3 & 0.06 & 1.5 \\
\hline KW106 & 10.16050 & 40.74816 & -5.5 & 3.11 & 3.96 & -0.9 & 0.26 & 2.7 \\
\hline KW107 & 10.16147 & 40.52894 & -4.5 & 2.67 & 3.36 & -0.1 & 0.08 & 1.1 \\
\hline KW110 & 10.16421 & 40.80204 & -4.9 & 3.02 & 3.74 & -0.4 & 0.05 & 3.3 \\
\hline KW112 & 10.17363 & 40.64115 & -8.2 & 3.88 & 5.71 & -1.2 & 0.51 & 6.0 \\
\hline KW113 & 10.17612 & 40.60131 & -6.5 & 3.38 & 4.67 & -0.7 & 0.06 & 6.5 \\
\hline KW114 & 10.17671 & 40.76094 & -8.7 & 2.00 & 4.56 & -0.3 & 1.16 & 2.9 \\
\hline KW115 & 10.17808 & 40.83946 & -5.3 & 2.05 & 3.27 & -0.3 & 0.06 & 2.7 \\
\hline KW119 & 10.18446 & 40.56793 & -7.2 & 1.38 & 3.55 & -0.6 & 0.48 & 2.8 \\
\hline KW120 & 10.18520 & 40.62048 & -8.8 & 1.94 & 4.57 & -0.7 & 1.07 & 2.6 \\
\hline KW121 & 10.18651 & 40.50836 & -7.2 & 1.99 & 3.93 & -0.6 & 0.65 & 3.4 \\
\hline KW122 & 10.18702 & 40.88564 & -6.5 & 2.58 & 4.06 & -0.6 & 0.41 & 1.6 \\
\hline KW123 & 10.19208 & 40.95922 & -5.4 & 2.30 & 3.51 & 0.1 & 0.03 & 2.3 \\
\hline KW124 & 10.19336 & 40.86136 & -6.7 & 1.81 & 3.65 & -0.8 & 0.30 & 2.5 \\
\hline KW125 & 10.19582 & 40.68280 & -5.7 & 3.42 & 4.38 & -0.6 & 0.04 & 3.2 \\
\hline KW126 & 10.19808 & 40.66878 & -8.9 & 0.70 & 3.64 & 0.1 & 1.37 & 3.2 \\
\hline KW127 & 10.19883 & 40.63281 & -6.2 & 3.16 & 4.31 & -0.4 & 0.24 & 5.9 \\
\hline KW129 & 10.19919 & 40.92626 & -5.8 & 2.38 & 3.71 & -0.0 & 0.05 & 4.5 \\
\hline KW130 & 10.20018 & 40.51726 & -5.7 & 3.32 & 4.27 & -0.8 & 0.07 & 2.9 \\
\hline KW131 & 10.20031 & 40.67784 & -6.9 & 2.31 & 4.04 & -0.6 & 0.77 & 2.6 \\
\hline KW132 & 10.20148 & 40.86623 & -7.0 & 1.34 & 3.51 & -0.1 & 0.23 & 1.3 \\
\hline KW133 & 10.20148 & 40.58504 & -6.8 & 2.31 & 3.99 & -0.5 & 0.10 & 1.9 \\
\hline KW134 & 10.20233 & 40.96013 & -6.7 & 2.50 & 4.12 & -0.2 & 0.33 & 2.1 \\
\hline KW135 & 10.20362 & 40.56563 & -6.5 & 1.83 & 3.64 & 0.0 & 0.19 & 6.5 \\
\hline KW136 & 10.20492 & 40.64867 & -5.5 & 2.93 & 3.90 & -1.0 & 0.07 & 1.1 \\
\hline KW137 & 10.20583 & 40.69227 & -7.5 & 3.36 & 5.01 & -1.0 & 0.18 & 4.2 \\
\hline
\end{tabular}


TABLE 1 - Continued

\begin{tabular}{|c|c|c|c|c|c|c|c|c|}
\hline ID & $\alpha_{\mathrm{J} 2000}$ & $\delta_{\mathrm{J} 2000}$ & $M_{V}^{\mathrm{a}}$ & $\log (t / \mathrm{Myr})^{\mathrm{b}}$ & $\log \left(m / m_{\odot}\right)^{\mathrm{c}}$ & {$[\mathrm{M} / \mathrm{H}]^{\mathrm{d}}$} & $E(B-V)^{\mathrm{e}}$ & $r_{h}{ }^{\mathrm{f}}$ \\
\hline KW139 & 10.21178 & 40.67737 & -6.0 & 3.20 & 4.36 & -0.5 & 0.39 & 3.3 \\
\hline KW140 & 10.21457 & 40.55768 & -7.7 & 2.31 & 4.33 & -0.6 & 0.47 & 6.7 \\
\hline KW141 & 10.21504 & 40.73508 & -8.7 & 0.84 & 3.81 & 0.6 & 0.65 & 0.8 \\
\hline KW142 & 10.21700 & 40.58377 & -5.8 & 1.85 & 3.30 & -0.5 & 0.32 & 0.7 \\
\hline KW143 & 10.21761 & 40.53484 & -4.9 & 2.66 & 3.49 & -0.5 & 0.06 & 3.1 \\
\hline KW144 & 10.21773 & 40.97823 & -6.6 & 2.78 & 4.21 & -1.0 & 0.33 & 1.8 \\
\hline KW145 & 10.21777 & 40.89900 & -6.5 & 3.62 & 5.04 & -0.1 & 0.07 & 8.8 \\
\hline KW146 & 10.22036 & 40.86882 & -6.2 & 3.20 & 4.33 & -1.3 & 0.35 & 4.2 \\
\hline KW147 & 10.22061 & 40.58877 & -7.1 & 3.75 & 5.11 & -1.9 & 0.34 & 5.8 \\
\hline KW149 & 10.22197 & 40.73255 & -6.5 & 0.70 & 2.66 & -0.0 & 0.62 & 1.0 \\
\hline KW150 & 10.22235 & 40.82742 & -6.1 & 3.81 & 4.87 & -0.6 & 0.42 & 3.8 \\
\hline KW151 & 10.22301 & 40.71887 & -5.9 & 2.37 & 3.70 & -0.3 & 0.51 & 1.2 \\
\hline KW152 & 10.22375 & 40.61417 & -5.4 & 3.97 & 4.62 & -1.4 & 0.24 & 1.1 \\
\hline KW153 & 10.22448 & 40.67137 & -4.6 & 2.75 & 3.49 & -0.1 & 0.06 & 1.2 \\
\hline KW154 & 10.22840 & 40.59210 & -5.5 & 2.64 & 3.68 & -0.8 & 0.14 & 1.6 \\
\hline KW155 & 10.22846 & 40.73891 & -5.4 & 3.37 & 4.19 & -0.8 & 0.09 & 2.0 \\
\hline KW156 & 10.23071 & 40.62244 & -5.9 & 1.90 & 3.47 & 0.2 & 0.49 & 2.1 \\
\hline KW158 & 10.23358 & 40.70630 & -7.0 & 2.20 & 3.99 & -0.6 & 0.62 & 1.6 \\
\hline KW159 & 10.23475 & 40.57826 & -5.3 & 2.34 & 3.42 & -0.6 & 0.21 & 1.2 \\
\hline KW160 & 10.23602 & 40.57370 & -5.9 & 2.48 & 3.77 & -0.6 & 0.06 & 3.6 \\
\hline KW161 & 10.23726 & 40.60918 & -7.3 & 2.45 & 4.29 & -0.5 & 0.83 & 2.2 \\
\hline KW164 & 10.23953 & 40.74093 & -7.8 & 4.01 & 5.58 & -1.9 & 0.59 & 1.8 \\
\hline KW165 & 10.23976 & 40.54874 & -6.2 & 1.58 & 3.32 & -0.2 & 0.25 & 1.9 \\
\hline KW166 & 10.24050 & 40.57272 & -5.3 & 1.43 & 2.84 & -1.0 & 0.06 & 0.7 \\
\hline KW168 & 10.24321 & 40.89079 & -9.3 & 0.70 & 3.81 & 0.4 & 1.49 & 2.3 \\
\hline KW169 & 10.24352 & 40.60981 & -6.9 & 1.50 & 3.58 & -0.0 & 0.39 & 0.8 \\
\hline KW170 & 10.24504 & 40.57345 & -6.2 & 1.85 & 3.59 & 0.3 & 0.27 & 0.9 \\
\hline KW171 & 10.24531 & 40.59666 & -10.6 & 4.00 & 6.72 & -1.6 & 0.14 & 3.7 \\
\hline KW172 & 10.24540 & 40.71805 & -6.4 & 3.83 & 5.23 & -0.0 & 0.51 & 4.9 \\
\hline KW173 & 10.24663 & 40.58450 & -5.9 & 1.86 & 3.34 & -0.7 & 0.26 & 2.4 \\
\hline KW174 & 10.24728 & 40.56506 & -5.3 & 2.38 & 3.48 & -0.4 & 0.18 & 1.1 \\
\hline KW176 & 10.25111 & 40.57447 & -7.4 & 0.90 & 3.09 & -0.3 & 0.81 & 1.4 \\
\hline KW177 & 10.26062 & 40.84083 & -5.0 & 2.12 & 3.14 & -0.5 & 0.19 & 1.0 \\
\hline KW178 & 10.26071 & 40.80432 & -6.8 & 4.02 & 5.51 & -0.0 & 0.34 & 3.2 \\
\hline KW180 & 10.26142 & 40.56423 & -6.1 & 2.03 & 3.56 & -0.5 & 0.14 & 1.8 \\
\hline KW181 & 10.26186 & 40.69644 & -5.9 & 2.84 & 4.00 & -0.9 & 0.36 & 1.9 \\
\hline KW182 & 10.26203 & 40.58293 & -6.5 & 1.93 & 3.65 & -0.4 & 0.14 & 1.2 \\
\hline KW183 & 10.26229 & 40.94328 & -5.6 & 4.09 & 5.11 & -0.0 & 0.08 & 9.7 \\
\hline KW184 & 10.26513 & 40.92902 & -6.3 & 3.30 & 4.45 & -1.4 & 0.36 & 5.8 \\
\hline KW185 & 10.26663 & 40.53928 & -6.8 & 2.26 & 3.96 & -0.5 & 0.43 & 9.2 \\
\hline KW186 & 10.26836 & 40.66409 & -5.2 & 2.98 & 3.84 & -0.5 & 0.12 & 1.0 \\
\hline KW187 & 10.26897 & 40.57899 & -6.5 & 1.23 & 3.22 & -0.1 & 0.20 & 1.2 \\
\hline KW188 & 10.27108 & 40.75779 & -6.1 & 2.46 & 3.85 & -0.4 & 0.38 & 2.1 \\
\hline KW189 & 10.27233 & 40.85868 & -5.2 & 2.23 & 3.32 & -0.5 & 0.07 & 1.5 \\
\hline KW190 & 10.27249 & 40.58317 & -6.8 & 1.54 & 3.60 & 0.1 & 0.21 & 0.9 \\
\hline KW191 & 10.27438 & 40.89986 & -6.0 & 2.65 & 3.90 & -0.8 & 0.24 & 4.3 \\
\hline KW192 & 10.27591 & 40.61567 & -6.1 & 1.82 & 3.41 & -0.8 & 0.35 & 3.7 \\
\hline KW193 & 10.27601 & 40.60383 & -5.3 & 2.39 & 3.51 & 0.1 & 0.04 & 3.6 \\
\hline KW194 & 10.27857 & 40.57474 & -6.9 & 1.80 & 3.70 & -0.8 & 0.24 & 2.8 \\
\hline KW195 & 10.28042 & 40.90625 & -4.7 & 3.02 & 3.66 & -0.4 & 0.04 & 1.8 \\
\hline KW196 & 10.28306 & 40.88364 & -6.9 & 4.01 & 5.32 & -0.8 & 0.11 & 2.7 \\
\hline KW197 & 10.28319 & 40.81240 & -6.5 & 1.89 & 3.61 & -0.7 & 0.45 & 5.4 \\
\hline KW198 & 10.28443 & 40.54785 & -7.5 & 2.10 & 4.16 & -0.4 & 0.51 & 4.5 \\
\hline KW199 & 10.28814 & 40.59810 & -8.4 & 3.84 & 5.81 & -0.7 & 0.09 & 2.2 \\
\hline KW200 & 10.29161 & 40.96981 & -7.0 & 3.19 & 4.66 & -0.9 & 0.16 & 5.7 \\
\hline KW201 & 10.29176 & 40.53308 & -4.8 & 2.50 & 3.40 & -0.0 & 0.04 & 3.3 \\
\hline KW203 & 10.29775 & 40.80557 & -6.7 & 1.34 & 3.44 & 0.2 & 0.29 & 1.6 \\
\hline KW204 & 10.29872 & 40.58854 & -5.3 & 2.32 & 3.42 & -0.4 & 0.16 & 1.4 \\
\hline KW205 & 10.29920 & 40.73882 & -5.0 & 3.02 & 3.85 & 0.1 & 0.04 & 4.5 \\
\hline KW206 & 10.29984 & 40.96378 & -6.9 & 2.22 & 3.94 & -0.6 & 0.62 & 4.0 \\
\hline KW207 & 10.30204 & 40.67383 & -6.9 & 2.09 & 3.88 & -0.6 & 0.66 & 2.9 \\
\hline KW208 & 10.30326 & 40.57155 & -7.7 & 1.75 & 4.01 & -0.3 & 0.24 & 2.9 \\
\hline KW209 & 10.30461 & 40.64758 & -4.9 & 2.76 & 3.60 & -0.0 & 0.06 & 2.6 \\
\hline KW210 & 10.30754 & 40.56614 & -7.3 & 2.24 & 4.19 & -0.4 & 0.06 & 9.3 \\
\hline KW211 & 10.31052 & 40.93089 & -9.3 & 4.00 & 6.19 & -1.6 & 0.17 & 2.2 \\
\hline KW212 & 10.31492 & 40.81869 & -5.2 & 0.84 & 2.09 & -0.2 & 0.09 & 1.3 \\
\hline KW213 & 10.31781 & 40.86897 & -5.0 & 2.93 & 3.69 & -0.9 & 0.03 & 2.1 \\
\hline KW214 & 10.31870 & 40.98434 & -8.2 & 4.10 & 5.78 & -2.2 & 0.19 & 1.7 \\
\hline KW215 & 10.31888 & 40.65087 & -6.5 & 1.84 & 3.57 & -0.8 & 0.14 & 1.4 \\
\hline KW216 & 10.31985 & 40.80730 & -4.5 & 2.53 & 3.30 & -0.0 & 0.05 & 2.5 \\
\hline KW217 & 10.32373 & 40.72907 & -5.8 & 2.49 & 3.75 & -0.5 & 0.32 & 1.4 \\
\hline KW218 & 10.32443 & 40.72654 & -7.9 & 0.70 & 3.16 & -0.3 & 1.09 & 2.9 \\
\hline KW219 & 10.32559 & 40.73372 & -6.7 & 2.79 & 4.28 & -0.5 & 0.20 & 4.0 \\
\hline KW221 & 10.32802 & 40.95444 & -8.1 & 3.93 & 6.03 & 0.1 & 0.35 & 1.7 \\
\hline KW223 & 10.32991 & 40.75117 & -6.8 & 3.98 & 5.20 & -1.4 & 0.26 & 1.3 \\
\hline KW224 & 10.33554 & 40.60722 & -4.3 & 1.84 & 2.70 & -0.4 & 0.02 & 1.1 \\
\hline
\end{tabular}


TABLE 1 - Continued

\begin{tabular}{|c|c|c|c|c|c|c|c|c|}
\hline ID & $\alpha_{\mathrm{J} 2000}$ & $\delta_{\mathrm{J} 2000}$ & $M_{V}^{\mathrm{a}}$ & $\log (t / \mathrm{Myr})^{\mathrm{b}}$ & $\log \left(m / m_{\odot}\right)^{\mathrm{c}}$ & {$[\mathrm{M} / \mathrm{H}]^{\mathrm{d}}$} & $E(B-V)^{\mathrm{e}}$ & $r_{h}{ }^{\mathrm{f}}$ \\
\hline KW225 & 10.33715 & 40.98462 & -7.2 & 3.95 & 5.60 & -0.1 & 0.17 & 1.7 \\
\hline KW226 & 10.33754 & 40.73732 & -6.0 & 2.30 & 3.71 & -0.4 & 0.41 & 1.4 \\
\hline KW227 & 10.33773 & 40.70366 & -6.4 & 2.40 & 3.92 & -0.4 & 0.39 & 2.0 \\
\hline KW228 & 10.34021 & 40.68455 & -6.2 & 2.23 & 3.72 & -0.5 & 0.28 & 1.6 \\
\hline KW229 & 10.34250 & 40.83295 & -6.5 & 3.79 & 4.89 & -1.9 & 0.48 & 4.8 \\
\hline KW230 & 10.34662 & 40.73745 & -4.4 & 2.88 & 3.47 & -0.4 & 0.05 & 1.4 \\
\hline KW231 & 10.34725 & 40.63251 & -5.4 & 2.42 & 3.54 & -0.1 & 0.24 & 3.4 \\
\hline KW232 & 10.35035 & 40.61306 & -6.8 & 1.98 & 3.79 & -0.5 & 0.06 & 8.2 \\
\hline KW233 & 10.35365 & 40.75562 & -5.0 & 2.92 & 3.85 & 0.3 & 0.07 & 1.3 \\
\hline KW234 & 10.35418 & 40.87940 & -4.6 & 3.04 & 3.77 & 0.3 & 0.07 & 2.2 \\
\hline KW235 & 10.35582 & 40.51488 & -6.5 & 3.82 & 5.07 & -0.5 & 0.56 & 2.3 \\
\hline KW236 & 10.35815 & 40.56083 & -5.5 & 2.58 & 3.69 & -0.4 & 0.20 & 3.8 \\
\hline KW239 & 10.36204 & 40.78528 & -6.6 & 0.95 & 3.03 & 0.1 & 0.61 & 3.1 \\
\hline KW240 & 10.36244 & 40.69372 & -6.8 & 2.06 & 3.81 & -0.4 & 0.31 & 1.8 \\
\hline KW242 & 10.36512 & 40.80364 & -6.3 & 2.02 & 3.60 & -0.4 & 0.48 & 0.9 \\
\hline KW243 & 10.36531 & 40.67627 & -5.0 & 3.01 & 3.75 & -0.5 & 0.04 & 1.1 \\
\hline KW244 & 10.36715 & 40.89721 & -9.1 & 3.86 & 6.22 & -0.3 & 0.04 & 1.8 \\
\hline KW245 & 10.37280 & 40.75469 & -6.1 & 2.59 & 3.95 & -0.3 & 0.14 & 4.2 \\
\hline KW246 & 10.37317 & 40.83282 & -7.8 & 1.88 & 4.19 & 0.1 & 0.94 & 3.2 \\
\hline KW247 & 10.37492 & 40.64060 & -5.7 & 1.76 & 3.25 & -0.2 & 0.20 & 2.8 \\
\hline KW248 & 10.37625 & 40.80908 & -5.1 & 2.47 & 3.49 & 0.1 & 0.04 & 4.8 \\
\hline KW249 & 10.37817 & 40.96697 & -10.5 & 0.70 & 4.30 & 0.4 & 1.46 & 13.5 \\
\hline KW250 & 10.37882 & 40.65230 & -5.7 & 3.40 & 4.26 & -1.6 & 0.24 & 2.9 \\
\hline KW251 & 10.37981 & 40.77018 & -5.7 & 2.56 & 3.79 & 0.1 & 0.03 & 2.2 \\
\hline KW252 & 10.38217 & 40.67094 & -6.5 & 1.49 & 3.34 & -0.5 & 0.69 & 2.1 \\
\hline KW253 & 10.38282 & 40.74846 & -4.6 & 2.46 & 3.27 & 0.0 & 0.05 & 2.8 \\
\hline KW254 & 10.38501 & 40.81791 & -5.0 & 3.07 & 3.86 & 0.0 & 0.06 & 3.0 \\
\hline KW255 & 10.38605 & 40.79066 & -5.6 & 2.41 & 3.65 & -0.1 & 0.32 & 2.9 \\
\hline KW256 & 10.38768 & 40.65660 & -5.6 & 1.89 & 3.25 & -0.5 & 0.28 & 1.6 \\
\hline KW257 & 10.38851 & 40.74583 & -6.3 & 2.27 & 3.76 & -0.5 & 0.55 & 3.9 \\
\hline KW258 & 10.38910 & 40.92238 & -9.9 & 0.70 & 4.05 & 0.4 & 1.66 & 3.4 \\
\hline KW259 & 10.38952 & 40.66234 & -4.7 & 2.57 & 3.39 & -0.1 & 0.05 & 1.8 \\
\hline KW260 & 10.38954 & 40.61910 & -5.4 & 2.38 & 3.57 & 0.0 & 0.03 & 2.3 \\
\hline KW261 & 10.38992 & 40.82127 & -8.5 & 0.70 & 3.48 & -0.0 & 1.39 & 2.1 \\
\hline KW262 & 10.39174 & 40.63835 & -5.2 & 2.73 & 3.72 & -0.1 & 0.05 & 5.0 \\
\hline KW263 & 10.39175 & 40.70641 & -7.0 & 2.39 & 4.16 & -0.4 & 0.68 & 2.8 \\
\hline KW264 & 10.39327 & 40.87083 & -5.8 & 2.39 & 3.73 & -0.1 & 0.26 & 1.9 \\
\hline KW265 & 10.39493 & 40.87377 & -6.6 & 1.46 & 3.38 & -0.2 & 0.59 & 1.1 \\
\hline KW266 & 10.39585 & 40.76342 & -6.0 & 3.17 & 4.24 & -0.6 & 0.06 & 8.6 \\
\hline KW267 & 10.39828 & 40.92745 & -6.7 & 4.03 & 5.50 & 0.0 & 0.37 & 2.9 \\
\hline KW268 & 10.39855 & 40.97107 & -5.4 & 4.01 & 4.62 & -1.9 & 0.16 & 3.5 \\
\hline KW269 & 10.39923 & 40.58348 & -7.4 & 1.08 & 3.43 & -0.9 & 0.99 & 1.7 \\
\hline KW270 & 10.40237 & 40.96336 & -5.4 & 2.60 & 3.70 & 0.1 & 0.05 & 1.0 \\
\hline KW271 & 10.40308 & 40.78978 & -9.3 & 0.84 & 3.75 & -0.2 & 0.92 & 5.4 \\
\hline KW 272 & 10.40315 & 40.84670 & -9.0 & 1.73 & 4.50 & -0.7 & 1.14 & 9.0 \\
\hline KW273 & 10.40363 & 40.79043 & -7.0 & 2.93 & 4.52 & -0.5 & 0.06 & 6.1 \\
\hline KW274 & 10.40468 & 40.94694 & -4.9 & 3.97 & 4.73 & 0.1 & 0.07 & 3.5 \\
\hline KW275 & 10.40531 & 40.68044 & -5.0 & 2.76 & 3.67 & 0.1 & 0.04 & 2.7 \\
\hline KW276 & 10.40555 & 40.88961 & -5.0 & 2.41 & 3.38 & -0.1 & 0.06 & 2.3 \\
\hline KW277 & 10.40689 & 40.81064 & -6.6 & 2.30 & 3.88 & -0.7 & 0.71 & 2.3 \\
\hline KW278 & 10.40818 & 40.79498 & -6.4 & 2.48 & 4.02 & -0.2 & 0.32 & 2.0 \\
\hline KW279 & 10.40857 & 40.56961 & -7.8 & 0.90 & 3.23 & -0.4 & 0.55 & 1.9 \\
\hline KW280 & 10.41025 & 40.97424 & -6.0 & 3.01 & 4.25 & 0.1 & 0.15 & 3.8 \\
\hline KW281 & 10.41050 & 40.82679 & -5.7 & 2.77 & 3.98 & 0.2 & 0.04 & 1.5 \\
\hline KW283 & 10.41167 & 40.79971 & -6.3 & 3.81 & 5.05 & -0.3 & 0.37 & 8.1 \\
\hline KW284 & 10.41180 & 40.68181 & -7.4 & 2.02 & 4.06 & -0.5 & 0.14 & 1.2 \\
\hline KW285 & 10.41559 & 40.94267 & -6.5 & 3.92 & 5.26 & -0.1 & 0.31 & 2.8 \\
\hline
\end{tabular}

Note. - ID number, R.A. \& Decl. (J2000) coordinates of the photometric aperture center in the USNO-B1.0 catalog system (degrees) according to Narbutis et al. (2008).

a Absolute magnitude, $M_{V}$, corrected for aperture correction.

$\mathrm{b}$ Age, $t$ in Myr, $\log (t / \mathrm{Myr})$.

C Mass, $m$ in solar mass units, $\log (m / m \odot)$.

d Metallicity, [M/H]

e Interstellar extinction in MW plus M31; color excess, $E(B-V)$.

f Average half-light radius in pc, $r_{h}=\left(r_{h}^{\mathrm{K}}+r_{h}^{\mathrm{E}}\right) / 2$, derived from the King and EFF models. 